$\mathrm{IPPP} / 03 / 23$

$\mathrm{DCPT} / 03 / 46$

SCIPP-04/15

hep-ph/0504050

April, 2005

\title{
Basis-independent methods for the two-Higgs-doublet model
}

\author{
Sacha Davidson ${ }^{1,2}$ and Howard E. Haber ${ }^{1,3}$ \\ ${ }^{1}$ Institute for Particle Physics Phenomenology \\ University of Durham, South Road, Durham DH1 3LE, U.K. \\ 2Institut de Physique Nucléaire de Lyon \\ Université Claude Bernard Lyon 1 \\ 4, rue Enrico Fermi, F-69622 Villeurbanne, France \\ ${ }^{3}$ Santa Cruz Institute for Particle Physics \\ University of California, Santa Cruz, CA 95064, U.S.A.
}

\begin{abstract}
In the most general two-Higgs-doublet model (2HDM), unitary transformations between the two Higgs fields do not change the functional form of the Lagrangian. All physical observables of the model must therefore be independent of such transformations (i.e., independent of the Lagrangian basis choice for the Higgs fields). We exhibit a set of basis-independent quantities that determine all tree-level Higgs couplings and masses. Some examples of the basis-independent treatment of 2HDM discrete symmetries are presented. We also note that the ratio of the neutral Higgs field vacuum expectation values, $\tan \beta$, is not a meaningful parameter in general, as it is basis-dependent. Implications for the more specialized 2HDMs (e.g., the Higgs sector of the MSSM and the so-called Type-I and Type-II 2HDMs) are explored.
\end{abstract}




\section{INTRODUCTION}

The Standard Model of electroweak physics, which posits a single hypercharge-one, $\mathrm{SU}(2)_{\mathrm{L}}$ doublet (complex) Higgs field, provides a extremely successful description of observed electroweak phenomena. Nevertheless, there are a number of motivations to extend the Higgs sector of this model by adding a second complex doublet Higgs field [1, 2, 3, 4, 5, [6]. Perhaps the best motivated of these extended models is the minimal supersymmetric extension of the Standard Model (MSSM) [7], which requires a second Higgs doublet (and its supersymmetric fermionic partners) in order to preserve the cancellation of gauge anomalies. The Higgs sector of the MSSM is a two-Higgs-doublet model (2HDM), which contains two Higgs supermultiplets $\widehat{H}_{u}$ and $\widehat{H}_{d}$, that are distinguished by the sign of their hypercharge [3, 4, 5]. This establishes an unambiguous "theoretical" basis for the Higgs sector of the Lagrangian. The structure of the MSSM Higgs sector is constrained by the supersymmetry, leading to numerous relations among Higgs masses and couplings. However, due to supersymmetry-breaking effects, all such relations are modified by loop-corrections, where the effects of supersymmetry-breaking can enter. Thus, one can describe the Higgs-sector of the (broken) MSSM by an effective field theory consisting of the most general two-Higgsdoublet model.

In a realistic model, the Higgs-fermion couplings must be chosen with some care [8, 9] to avoid flavor-changing-neutral-currents (FCNC). The 2HDM can be classified by how this issue is addressed. In type-I models [10, 11], there exists a basis choice in which only one of the Higgs fields couples to the Standard Model fermions. In type-II models [11, 12], there exists a basis choice in which one Higgs field couples to up-type quarks, and the other Higgs field couples to down-type quarks. Type-III models [13] allow both Higgs fields to couple to all the Standard Model fermions; such a model is phenomenologically viable only if the resulting FCNC-couplings are sufficiently small.

From a phenomenological bottom-up perspective, it is important to study the properties of the most general 2HDM [14] without imposing any special relations among the tree-level parameters. The 2HDM Lagrangian depends on two identical hypercharge-one, SU(2) $)_{\mathrm{L}}$ doublet Higgs fields, $\Phi_{1}$ and $\Phi_{2}$. If $\Phi_{1}$ and $\Phi_{2}$ couple identically to all other fields (fermions and gauge bosons), they are only distinguished by the scalar interactions contained in the scalar Higgs potential. Consequently, one is free to choose an arbitrary basis in the Lagrangian 
for the two dimensional space of Higgs fields [15], corresponding to two arbitrary orthogonal linear combinations of $\Phi_{1}$ and $\Phi_{2}$. The couplings and mass matrix elements will depend on this basis choice. In particular, the important parameter of the MSSM, $\tan \beta \equiv\left\langle H_{u}^{0}\right\rangle /\left\langle H_{d}^{0}\right\rangle$, is not a physical parameter of the most general 2HDM since its definition refers to a specific basis choice for the Higgs fields. The aim of this paper is to establish a basis-independent formalism for analyzing the most general 2HDM. This provides a framework for expressing any physical observable (e.g., Higgs masses and couplings) in a form that is independent of the basis choice.

It is often phenomenologically desirable to express the parameters of a field theory as functions of basis-independent physical observables [16, 17, 18]. This procedure has various advantages: it leads to parameters that are uniquely defined, gauge invariant and typically well behaved under renormalization. One simple and straightforward alternative is to choose a basis and consistently calculate there. However, results expressed in terms of basisdependent Lagrangian parameters are meaningless without identifying the basis, and the comparison with results computed in a different basis choice is often difficult. This situation can be avoided by constructing quantities that are invariant under arbitrary basis transformations in the space of fields, and expressing the fundamental Lagrangian parameters in terms of these "invariants". We wish to draw attention to this procedure in the 2HDM, where one must specify the basis for the scalar fields before defining the Higgs potential parameters, the Higgs-fermion Yukawa couplings and $\tan \beta$.

In the 2HDM, we define "invariants" to be quantities that are scalar under arbitrary unitary transformations among the two Higgs fields in the Lagrangian [6, 15, 19, 20]. All physical observables can be expressed in terms of invariants. The parameters that appear in the Lagrangian with respect to a generic basis of Higgs fields are not physical. Nevertheless, even in the most general model, certain specific basis choices are singled out, and the corresponding Lagrangian parameters become meaningful. Henceforth, we shall designate such parameters as "physical parameters." For example, in the CP-conserving 2HDM, the Lagrangian parameters in the basis corresponding to the neutral CP-even Higgs mass eigenstates are physical parameters [21]. That is, any coupling or mixing angle in the mass eigenstate basis can be expressed in terms of invariants. One particularly useful class of bases is the so-called Higgs basis [9, 15, 19, 22, 23] in which only one of the two scalar fields exhibits a non-zero vacuum expectation value. We shall demonstrate that the Lagrangian 
parameters with respect to the Higgs basis are closely related to physical parameters.

In this paper, we construct invariants in two ways. In the body of the paper, we combine covariant tensors constructed from the Higgs potential parameters and the vacuum expectation values to obtain scalar quantities. This elegant approach is rather abstract, so in the appendices we explicitly construct eigenbases corresponding to the orthonormal eigenvectors of various physically relevant second-ranked tensors. By contracting these eigenvectors with the Higgs potential parameter tensors, one can identify which combinations are invariant and correspond to physical parameters.

In section III, the scalar potential is expressed in a covariant notation with respect to the $\mathrm{U}(2)$ transformations between the two Higgs fields, and we introduce a set of invariants that govern the model. These invariants depend on the Higgs potential parameter tensors and a matrix $V$ constructed from the vacuum expectations values. The eigenvectors of $V$ form an orthonormal basis that defines the Higgs basis. A review of the 2HDM in a generic basis and in the Higgs basis can be found in Appendix A, where we also exhibit explicit relations among the corresponding Higgs potential parameters. In Appendix B we explicitly combine eigenvectors of $V$ with Higgs potential parameter tensors to obtain quantities that depend linearly on the Higgs potential parameters. We show that some of these are invariant with respect to $\mathrm{U}(2)$ transformations and hence "physical", whereas others have undetermined phases. In section III we construct invariants that vanish when the discrete symmetries $\Phi_{1} \rightarrow \Phi_{1}, \Phi_{2} \rightarrow-\Phi_{2}$ and CP-symmetry, respectively, are realized. The relation of the $\Phi_{1} \rightarrow \Phi_{1}, \Phi_{2} \rightarrow-\Phi_{2}$ discrete symmetry to the permutation symmetry $\Phi_{1} \leftrightarrow \Phi_{2}$ is discussed in Appendix C. In sections IV and D. we restrict our analysis to the CP-conserving 2HDM scalar potential. We first construct the physical Higgs boson couplings to vector bosons and the Higgs self-couplings in terms of basis-independent parameters. We then study the Higgs-fermion interactions in the 2HDM, using a choice of basis motivated by the model to compute the physical Higgs-fermion couplings in terms of invariant couplings. In doing so, new $\tan \beta$-like parameters arise that are directly defined in terms of physical Yukawa couplings. Both CP-conserving and CP-violating Higgs-fermion interactions are treated. Finally, a brief summary is provided in section VI. 


\section{BASIS-INDEPENDENT ANALYSIS OF THE 2HDM}

The most general two-Higgs-doublet extension of the Standard Model [4, 14, 24] is a theory of two identical hypercharge-one complex doublet scalar fields $\Phi_{1}$ and $\Phi_{2}$. We shall denote the complex two-dimensional space spanned by these two fields as the Higgs flavor space. The canonically normalized (gauge-covariant) kinetic energy terms of the scalar fields are invariant under arbitrary global U(2) transformations in this flavor space. Thus, we are free to redefine our two scalar fields by making an arbitrary $\mathrm{U}(2)$ transformation. A specific choice of fields will be called a choice of basis. Since physical observables are independent of this choice of basis, it is desirable to formulate the Higgs sector of the theory in a basisindependent manner.

Nearly all discussions of Higgs physics employ a specific basis choice. In the generic basis, one expands the Higgs fields around their vacuum expectation values $\left\langle\Phi_{a}^{0}\right\rangle=v_{a} / \sqrt{2}(a=$ $1,2)$, and the parameter $\tan \beta \equiv v_{2} / v_{1}$ plays an important role in the Higgs phenomenology. However, this parameter cannot be meaningful in general, as it is basis-dependent. In fact, this parameter disappears completely if one transforms to the Higgs basis, where the Higgs vacuum expectation value resides solely in one of the two Higgs doublets. ${ }^{1}$ This suggests that the Higgs basis is special. In this section, we shall demonstrate that the parameters that appear in the Higgs basis are closely related to basis-independent quantities.

The scalar Higgs potential of the 2HDM can be written in terms of the two complex hypercharge-one, $\mathrm{SU}(2)_{L}$ doublet scalar fields, following ref. [6], as:

$$
\mathcal{V}=Y_{a \bar{b}} \Phi_{\bar{a}}^{\dagger} \Phi_{b}+\frac{1}{2} Z_{a \bar{b}} c \bar{d}\left(\Phi_{\bar{a}}^{\dagger} \Phi_{b}\right)\left(\Phi_{\bar{c}}^{\dagger} \Phi_{d}\right)
$$

where the indices $a, \bar{b}, c$ and $\bar{d}$ run over the two-dimensional Higgs flavor space and

$$
Z_{a \bar{b} c \bar{d}}=Z_{c \bar{d} a \bar{b}}
$$

Hermiticity of $\mathcal{V}$ implies that

$$
\begin{aligned}
Y_{a \bar{b}} & =\left(Y_{b \bar{a}}\right)^{*}, \\
Z_{a \bar{b} c \bar{d}} & =\left(Z_{b \bar{a} d \bar{c}}\right)^{*} .
\end{aligned}
$$

\footnotetext{
1 The generic basis and Higgs basis are reviewed in Appendix A In particular, eqs. (A13)-(A22) provide the relations between the Higgs potential parameters in the generic and Higgs bases.
} 
We can match the standard 2HDM notation given in eq. (A1) by making the following identifications:

$$
\begin{array}{ll}
Y_{11}=m_{11}^{2}, & Y_{12}=-m_{12}^{2}, \\
Y_{21}=-\left(m_{12}^{2}\right)^{*}, & Y_{22}=m_{22}^{2},
\end{array}
$$

and

$$
\begin{array}{ll}
Z_{1111}=\lambda_{1}, & Z_{2222}=\lambda_{2}, \\
Z_{1122}=Z_{2211}=\lambda_{3}, & Z_{1221}=Z_{2112}=\lambda_{4}, \\
Z_{1212}=\lambda_{5}, & Z_{2121}=\lambda_{5}^{*}, \\
Z_{1112}=Z_{1211}=\lambda_{6}, & Z_{1121}=Z_{2111}=\lambda_{6}^{*}, \\
Z_{2212}=Z_{1222}=\lambda_{7}, & Z_{2221}=Z_{2122}=\lambda_{7}^{*} .
\end{array}
$$

We assume that the vacuum respects the electromagnetic gauge symmetry. That is, the vacuum expectation values of $\Phi_{1}$ and $\Phi_{2}$ are assumed to be aligned in $S U(2)_{L}$ space, and we follow the standard convention (after using the appropriate $\mathrm{SU}(2)_{\mathrm{L}}$ transformation) in writing:

$$
\left\langle\Phi_{a}\right\rangle=\frac{v}{\sqrt{2}}\left(\begin{array}{c}
0 \\
\widehat{v}_{a}
\end{array}\right),
$$

where $\widehat{v}_{a}$ is a vector of unit norm. Taking the derivative of eq. (11) with respect to $\Phi_{b}$, and setting $\left\langle\Phi_{a}^{0}\right\rangle=v_{a} / \sqrt{2}$, we find the covariant form for the scalar potential minimum conditions:

$$
\widehat{v}_{\bar{a}}^{*}\left[Y_{a \bar{b}}+\frac{1}{2} v^{2} Z_{a \bar{b} c \bar{d}} \widehat{v}_{\bar{c}}^{*} \widehat{v}_{d}\right]=0
$$

Under the flavor $\mathrm{SU}(2)$, the two Higgs doublets fields transform as $\Phi_{a} \rightarrow U_{a \bar{b}} \Phi_{b}$ (and $\left.\Phi_{\bar{a}}^{\dagger}=\Phi_{\bar{b}}^{\dagger} U_{b \bar{a}}^{\dagger}\right)$, where $U_{b \bar{a}}^{\dagger} U_{a \bar{c}}=\delta_{b \bar{c}}$ and det $U=1$. Likewise, the tensors $Y$ and $Z$ transform covariantly: $Y_{a \bar{b}} \rightarrow U_{a \bar{c}} Y_{c \bar{d}} U_{d \bar{b}}^{\dagger}$ and $Z_{a \bar{b} c \bar{d}} \rightarrow U_{a \bar{e}} U_{f \bar{b}}^{\dagger} U_{c \bar{g}} U_{h \bar{d}}^{\dagger} Z_{e \bar{f} g \bar{h}}$. The use of barred indices is convenient for keeping track of which indices transform with $U$ and which transform with $U^{\dagger}$. For example, in this notation, $v_{\bar{a}}^{*}=\left(v_{a}\right)^{*}$, which makes the starred superscript on $v_{\bar{a}}$ superfluous (although we will maintain it for the sake of additional clarity). These $\mathrm{SU}(2)$ transformations are symmetries of the physics (not of the Lagrangian), in the sense that physical observables must not depend on the arbitrary basis choice made in the Lagrangian. 
The flavor-SU(2) group of transformations can be enlarged to $\mathrm{U}(2) .{ }^{2}$ In particular, the transformation laws noted above continue to hold where $U$ is a $U(2)$ matrix (and the condition $\operatorname{det} U=1$ is removed). It should be noted that $\mathrm{U}(2) \cong \mathrm{SU}(2) \times \mathrm{U}(1)_{\mathrm{Y}}$, where the (global) hypercharge $\mathrm{U}(1)_{\mathrm{Y}}$ transformation is a symmetry of the Lagrangian, and thus has no effect on the parameters of the Higgs potential, $Y_{a \bar{b}}$ and $Z_{a \bar{b} c \bar{d}}$. It is useful to keep track of the $\mathrm{U}(1)_{Y}$ transformation properties of various quantities because physical observables must also be $\mathrm{U}(1)_{Y}$-invariant. Thus, we shall focus on $\mathrm{U}(2)$-invariant scalars, keeping in mind the distinction that $\mathrm{U}(1)_{Y}$ is a symmetry of the Lagrangian, whereas the flavor-SU(2) transformations change the parameters of the Lagrangian,

We shall construct various $\mathrm{U}(2)$-invariants below that depend on the Higgs potential parameters $Y_{a \bar{b}}$ and $Z_{a \bar{b} c \bar{d}}$ and the vacuum expectation values $\widehat{v}_{a}$. In particular, any $\mathrm{U}(2)$ invariant that depends on the vacuum expectation values must be a function of $\widehat{v}_{a} \widehat{v}_{\bar{b}}^{*}$. Thus we follow ref. [6] in defining the hermitian matrix ${ }^{3}$

$$
V_{a \bar{b}} \equiv \widehat{v}_{a} \widehat{v}_{\bar{b}}^{*}
$$

The matrix $V$ transforms covariantly with respect to $\mathrm{U}(2)$. Note that $V$ possesses two eigenvalues, 1 and 0, corresponding to orthonormal eigenvectors $\widehat{v}_{a}$ and

$$
\widehat{w}_{a} \equiv-\epsilon_{a b} \widehat{v}_{\bar{b}}^{*},
$$

with the inverse relation $\widehat{v}_{\bar{a}}^{*}=\epsilon_{\bar{a} \bar{b}} w_{b}$. Here, orthogonality with respect to the complex twodimensional Higgs flavor space means that $\widehat{v}_{\bar{a}} \widehat{w}_{a}^{*}=0$. In defining the unit vector $\widehat{w}_{a}$, we have introduced the tensors $\epsilon_{a b}$ and $\epsilon_{\bar{a} \bar{b}}$, with $\epsilon_{12}=-\epsilon_{21}=1$ and $\epsilon_{11}=\epsilon_{22}=0 .{ }^{4}$ The normalized eigenvectors are defined only up to an arbitrary phase, which implies that invariant quantities can only depend on $\widehat{v}$ and $\widehat{w}$ via the combination $V_{a \bar{b}}$, as noted above. In particular,

$$
W_{a \bar{b}} \equiv \widehat{w}_{a} \widehat{w}_{\bar{b}}^{*}=\delta_{a \bar{b}}-V_{a \bar{b}}
$$

${ }^{2}$ The flavor-U(2) transformations were first applied to the $2 \mathrm{HDM}$ in ref. 15]. A nice textbook discussion is given in ref. [6]. These transformations have recently been exploited by ref. [20] in a study of the CP-violating 2HDM.

${ }^{3} V_{a \bar{b}}$ is not an independent tensor, since it depends on $Y$ and $Z$ via the scalar potential minimum conditions [eq. (8)]. The latter can be rewritten as: $(V Y)_{a \bar{b}}+\frac{1}{2} v^{2} Z_{e \bar{b} c \bar{d}} V_{a \bar{e}} V_{d \bar{c}}=0$. Nevertheless, there is no simple closed-form formula for $V_{a \bar{b}}$ in terms of $Y_{a \bar{b}}$ and $Z_{a \bar{b} c \bar{d}}$. Consequently, we shall construct invariants that depend explicitly on $V_{a \bar{b}}$ in what follows.

${ }^{4}$ Note that $\delta_{a \bar{b}}$ is a flavor-U(2)-invariant tensor, whereas $\epsilon_{a b}$ and $\epsilon_{\bar{a} \bar{b}}$ are only flavor-SU(2)-invariant tensors. The identity $\epsilon_{a b} \epsilon_{\bar{c} \bar{d}}=\delta_{a \bar{c}} \delta_{b \bar{d}}-\delta_{a \bar{d}} \delta_{b \bar{c}}$ relates the two tensors. 
so that $W_{a \bar{b}}$ is not an independent tensor. Eq. (11) and the following results imply that $V$ and $W$ are projection operators:

$$
V^{2}=V, \quad W^{2}=W, \quad V W=W V=0 .
$$

Since the tensors $V_{a \bar{b}}, Y_{a \bar{b}}$ and $Z_{a \bar{b} c \bar{d}}$ exhibit tensorial properties with respect to global U(2) rotations in the Higgs flavor space, one can easily construct invariants with respect to $\mathrm{U}(2)$ by forming $\mathrm{U}(2)$-scalar quantities. Combinations of covariant tensors can be represented diagrammatically. The tensors $V_{a \bar{b}}, Y_{a \bar{b}}$ and $Z_{a \bar{b} c \bar{d}}$ can be depicted by two and four-point "vertices", with incoming (outgoing) lines corresponding to unbarred (barred) indices, as shown in Fig. 1.
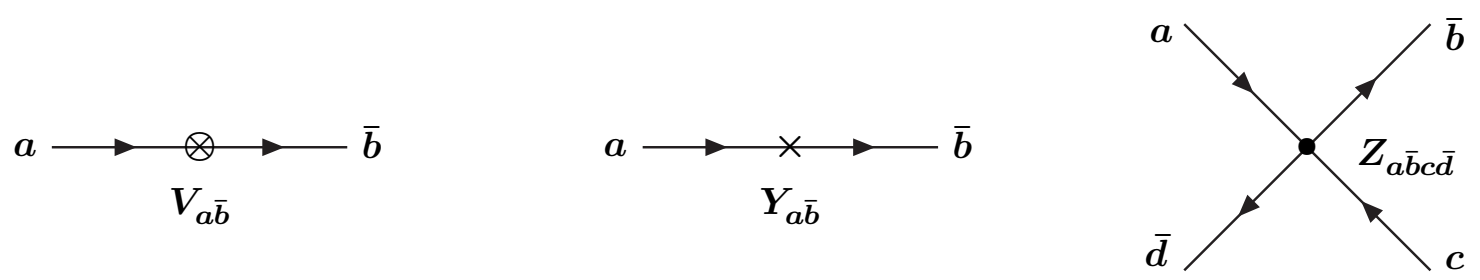

FIG. 1: Diagrammatic representation of covariant tensors. The two point vertices $V_{a \bar{b}}$ and $Y_{a \bar{b}}$ are indicated by the symbols $\otimes$ and $\times$, respectively. The four-point vertex $Z_{a \bar{b} c \bar{d}}$ is depicted by four incoming line segments (meeting at the vertex point) where the indices appear in clockwise order. Unbarred (barred) indices are represented by incoming (outgoing) directed line segments.

As noted above, $\delta_{a \bar{b}}$ is the only $\mathrm{U}(2)$-invariant tensor that can be used to contract a pair of indices. Hence, one can only contract an unbarred index with a barred index (or vice versa). For example,

$$
Z_{a \bar{d}}^{(1)} \equiv \delta_{b \bar{c}} Z_{a \bar{b} c \bar{d}}=Z_{a \bar{b} b \bar{d}}, \quad \quad Z_{c \bar{d}}^{(2)} \equiv \delta_{b \bar{a}} Z_{a \bar{b} c \bar{d}}=Z_{a \bar{a} c \bar{d}}
$$

Using the vertex rules given in Fig. 1, the two tensors of eq. (13) can be depicted diagrammatically as the one-loop bubble diagrams shown in Fig. 2. Similarly, U(2)-invariant quantities can be represented by "vacuum" diagrams with no external lines.

We now turn to the task of constructing independent invariant (scalar) combinations of Higgs potential parameters. Such invariant quantities can be directly related to physical quantities which must be basis-independent. We may combine the tensors $Y_{a \bar{b}}, Z_{a \bar{b} c \bar{d}}$, and $V_{a \bar{b}}$ to create scalar quantities by summing over pairs of indices (following the rule that the 


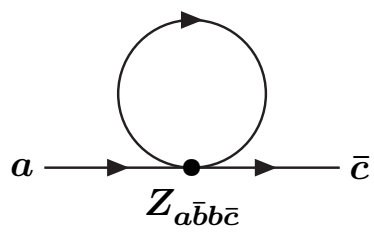

(a) $Z_{a \bar{c}}^{(1)}$

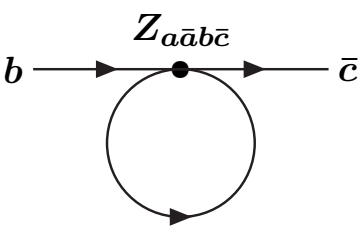

(b) $Z_{b \bar{c}}^{(2)}$

FIG. 2: The one-loop bubble diagrams corresponding to (a) $Z_{a \bar{c}}^{(1)}=Z_{a \bar{b} b \bar{c}}$ and (b) $Z_{b \bar{c}}^{(2)}=Z_{a \bar{a} b \bar{c}}$. These two diagrams are distinguished, since the $Z$-vertex must be read in a clockwise fashion.

summed index pairs must contain one unbarred and one barred index). It is also convenient to employ $W_{a \bar{b}} \equiv \delta_{a \bar{b}}-V_{a \bar{b}}$ introduced above. We can exhibit thirteen invariants that depend on the Higgs potential parameters and the vacuum expectation value by defining six real flavor-U(2)-invariants (denoted below by $Y_{1,2}$ and $Z_{1,2,3,4}$ ) and four complex index-free quantities, denoted by $Y_{3}$ and $Z_{5,6,7}$, which are constructed from $Y_{a \bar{b}}, Z_{a \bar{b} c \bar{d}} v_{a}$ and $w_{a}$. These quantities are invariant under the Higgs flavor-SU(2) transformations, but are not invariant with respect to $\mathrm{U}(1)_{\mathrm{Y}}$ as shown in Appendix B, From these four complex $\mathrm{SU}(2)$ - "invariants", we may extract seven flavor-U(2) invariants consisting of the magnitudes and relative phases of these four complex quantities.

The simplest invariants that can be constructed with $Y, V$ and $W$ are given by

$$
\begin{aligned}
Y_{1} & \equiv \operatorname{Tr}(Y V), \\
Y_{2} & \equiv \operatorname{Tr}(Y W), \\
\left|Y_{3}\right|^{2} & \equiv \operatorname{Tr}(V Y W Y) .
\end{aligned}
$$

Likewise, the simplest invariants that can be constructed with $Z, V$ and $W$ are given by:

$$
\begin{aligned}
Z_{1} & \equiv Z_{a \bar{b} c \bar{d}} V_{b \bar{a}} V_{d \bar{c}}, \\
Z_{2} & \equiv Z_{a \bar{b} c \bar{d}} W_{b \bar{a}} W_{d \bar{c}}, \\
Z_{3} & \equiv Z_{a \bar{b} c \bar{d}} V_{b \bar{a}} W_{d \bar{c}}, \\
Z_{4} & \equiv Z_{a \bar{b} \bar{c} \bar{d}} W_{d \bar{a}} V_{b \bar{c}}, \\
\left|Z_{5}\right|^{2} & \equiv Z_{a \bar{c} \bar{c} \bar{d}} Z_{e \bar{g} \bar{g} \bar{h}} V_{f \bar{a}} W_{b \bar{e}} V_{h \bar{c}} W_{d \bar{g}}, \\
\left|Z_{6}\right|^{2} & \equiv Z_{a \bar{b} c \bar{d} \bar{d}} Z_{e \bar{g} \bar{g} \bar{h}} V_{f \bar{a}} V_{b \bar{e}} V_{h \bar{c}} W_{d \bar{g}}, \\
\left|Z_{7}\right|^{2} & \equiv Z_{a \bar{b} c \bar{d} \bar{d}} Z_{e \bar{g} \bar{g} \bar{h}} V_{f \bar{a}} W_{b \bar{e}} W_{h \bar{c}} W_{d \bar{g}} .
\end{aligned}
$$


The remaining three invariants correspond to three relative phases of $Y_{3}$ and $Z_{5,6,7}$. One possible choice is:

$$
\begin{aligned}
Y_{3}^{2} Z_{5}^{*} & \equiv(V Y W)_{b \bar{a}}(V Y W)_{d \bar{c}} Z_{a \bar{b} c \bar{d}} \\
Y_{3} Z_{6}^{*} & =(V Y W)_{b \bar{c}} V_{d \bar{a}} Z_{a \bar{b} c \bar{d}} \\
Y_{3} Z_{7}^{*} & =(V Y W)_{b \bar{c}} W_{d \bar{a}} Z_{a \bar{b} c \bar{d}}
\end{aligned}
$$

Using the rules of Fig. 1, each of the invariants above is easily represented by a simple vacuum diagram (or sum of such diagrams). Using these diagrammatic techniques, one can easily show that no additional independent invariants exist that are linear or quadratic in the Higgs potential parameters. Further higher-order invariants could be constructed containing additional powers of $Y, V$ or $W$. However, we will argue below, and prove in Appendix B that any higher order invariant can be expressed in terms of those given in eqs. (14)-(26).

The invariants given in eqs. (14)-(26) are particularly simple in the Higgs basis [see Appendix $\mathrm{A}$, where $V$ and $W$ are given by:

$$
V=\left(\begin{array}{ll}
1 & 0 \\
0 & 0
\end{array}\right), \quad W=\left(\begin{array}{ll}
0 & 0 \\
0 & 1
\end{array}\right) .
$$

Following Appendix A, we denote the Higgs potential parameters in the Higgs basis by $M_{i j}^{2}$ $(i, j=1,2)$ and $\Lambda_{k}(k=1, \ldots, 7)$. After inserting the Higgs basis forms for $V$ and $W$ into eqs. (14)-(26), we obtain:

$$
Y_{1}=M_{11}^{2}, \quad Y_{2}=M_{22}^{2}, \quad Z_{i}=\Lambda_{i}(i=1,2,3,4)
$$

for the real Higgs basis parameters and

$$
\begin{aligned}
& \left|Y_{3}\right|^{2}=\left|M_{12}^{2}\right|, \quad\left|Z_{i}\right|^{2}=\left|\Lambda_{i}\right|^{2} \quad(i=5,6,7), \\
& Y_{3}^{2} Z_{5}^{*}=\left[M_{12}^{2}\right]^{2} \Lambda_{5}^{*}, \quad Y_{3} Z_{i}^{*}=-M_{12}^{2} \Lambda_{i}^{*} \quad(i=6,7),
\end{aligned}
$$

for the complex Higgs basis parameters.

The form of eq. (27) implies that $V$ and $W$ serve as projection operators for the Higgs basis field invariants $H_{1}^{\dagger} H_{1}$ and $H_{2}^{\dagger} H_{2}$. In particular, in the diagrammatic representation of invariants, the insertion of $V$ projects out $H_{1}$ and the insertion of $W$ projects out $H_{2}$. Thus, each of the lines of the vacuum diagrams corresponding to the invariants listed in eqs. (14)-(26) can be identified with one of the two fields $H_{1}$ and $H_{2}$ of the Higgs basis. All 
such vacuum diagrams must contain an equal number of fields and their complex conjugates (corresponding to ingoing and outgoing lines). Hence, there are no complex U(2)-invariants that are linear in the scalar potential parameters.

The scalar minimum conditions can be rewritten in terms of the U(2)-invariants introduced above. First we multiply eq. (을 by $\widehat{v}_{b}$ to obtain one of the minimum conditions:

$$
Y_{1}=-\frac{1}{2} Z_{1} v^{2}
$$

A second minimum condition can be obtained by multiplying eq. (8) by $\widehat{w}_{b}$. Using eqs. (B4) and ((B) $)$, it follows that $Y_{3}=-\frac{1}{2} Z_{6} v^{2}$, which can be rewritten in terms of $\mathrm{U}(2)$-invariant quantities as

$$
\left|Y_{3}\right|^{2}=\frac{1}{4}\left|Z_{6}\right|^{2} v^{4}, \quad Y_{3} Z_{6}^{*}=-\frac{1}{2}\left|Z_{6}\right|^{2} v^{2} .
$$

Consequently, after imposing the scalar minimum conditions, two of the thirteen invariants above can be eliminated via eq. (31), leaving eleven independent invariants. ${ }^{5}$ This corresponds precisely to the number of parameter degrees of freedom of the 2HDM, as discussed in Appendix $\mathrm{A}$

Having enumerated the independent invariants, one can begin to relate them to physical observables of the theory. One consequence of the analysis relating invariants to the Higgs potential parameters in the Higgs basis is that the parameter $\tan \beta$ is redundant (as it does not appear in the Higgs basis description of the 2HDM). More precisely, in the general 2HDM, $\tan \beta$ is a basis-dependent quantity. This means that physical quantities (e.g., Higgs masses and physical couplings) cannot depend on the (unphysical) angle between the two vacuum expectation values. Physical quantities can depend on the angle between the vacuum expectation value $\widehat{v}_{a}$ and another direction in the Higgs flavor space picked out by an interaction (e.g. a mass matrix eigenvector). We illustrate this explicitly in section IV] This suggests a procedure to provide a meaningful definition of $\tan \beta$. For example, even in the most general 2HDM, the Higgs potential depends on tensorial quantities, which can be used to define various matrices. The two eigenvectors of these matrices can be used as basis vectors which defines a basis that generally differs from the Higgs basis, and thus can be used to define $\tan \beta$. In a CP-invariant theory, an obvious choice would be the CP-even mass eigenstate basis.

\footnotetext{
${ }^{5}$ Since we count $v^{2}$ as one of the parameter degrees of freedom, the application of the scalar potential minimum conditions does not affect the overall counting of degrees of freedom.
} 
Other possible matrices, whose eigenvectors can be used to define a new basis, include: $Y_{a \bar{b}}, Z_{a \bar{b}}^{(1)}$ and $Z_{a \bar{b}}^{(2)}$. However, in such cases there is a two-fold ambiguity in the definition of $\tan \beta$ corresponding to the interchange of the two identical scalar fields. As an example, consider a definition of $\tan \beta$ corresponding to a basis in which $Y_{12}=0$. This is the basis spanned by the eigenvectors of $Y$. In this basis, we define $\left|\widehat{v}_{1}\right|=\cos \beta$ and $\left|\widehat{v}_{2}\right|=\sin \beta$ (where $0 \leq \beta \leq \pi / 2$ ). A simple computation in the $Y_{12}=0$ basis yields:

$$
\begin{aligned}
2 \operatorname{Tr}(V Y)-\operatorname{Tr} Y & =\left(Y_{11}-Y_{22}\right) \cos 2 \beta, \\
2 \operatorname{Tr}\left(Y^{2}\right)-(\operatorname{Tr} Y)^{2} & =\left(Y_{11}-Y_{22}\right)^{2} .
\end{aligned}
$$

Thus, we arrive at one possible invariant definition of $\cos ^{2} 2 \beta$ :

$$
\cos ^{2} 2 \beta=\frac{[2 \operatorname{Tr}(V Y)-\operatorname{Tr} Y]^{2}}{2 \operatorname{Tr}\left(Y^{2}\right)-(\operatorname{Tr} Y)^{2}} .
$$

The sign of $\cos 2 \beta$ is ambiguous, and the ambiguity corresponds to $\beta \rightarrow \pi / 2-\beta$ (i.e., the interchange of the two Higgs fields).

\section{BASIS-INDEPENDENT DESCRIPTION OF DISCRETE SYMMETRIES}

In section III, we assumed that the scalar Higgs potential takes on the most general possible form. However, in some two-Higgs doublet models, discrete symmetries are imposed that constrain the structure of the Higgs potential. Typically, the discrete symmetries are formulated with respect to a particular basis, in which case the symmetry is manifest. In a generic basis, the discrete symmetry is of course still present, but in most cases it is well disguised and not immediately evident. Hence, for a given discrete symmetry, it is desirable to establish a basis-independent characterization. In particular, it is especially useful to establish basis-independent conditions that depend only on the $Y$ and $Z$ tensors, i.e. they do not require a determination of the vacuum expectation values via the scalar potential minimum conditions. A number of examples will be presented in sections ஹB and IIC, in which we identify invariant conditions for discrete symmetries. These conditions can be evaluated in the Higgs basis, and provide a connection to related results first obtained in ref. [23].

However, in some special regions of the Higgs potential parameter space many of the invariant conditions that we obtain are automatically satisfied (due to the enhanced symmetry of the parameter region). This complicates the search for invariant conditions for 
the discrete symmetries, so we first examine the nature of these exceptional regions of the parameter space.

\section{A. An exceptional region of the Higgs potential parameter space}

Consider the explicit forms of $Z^{(1)}$ and $Z^{(2)}$ defined in eq. (13):

$$
Z^{(1)}=\left(\begin{array}{cc}
\lambda_{1}+\lambda_{4} & \lambda_{6}+\lambda_{7} \\
\lambda_{6}^{*}+\lambda_{7}^{*} & \lambda_{2}+\lambda_{4}
\end{array}\right), \quad Z^{(2)}=\left(\begin{array}{cc}
\lambda_{1}+\lambda_{3} & \lambda_{6}+\lambda_{7} \\
\lambda_{6}^{*}+\lambda_{7}^{*} & \lambda_{2}+\lambda_{3}
\end{array}\right)
$$

Note that $Z^{(1)}$ and $Z^{(2)}$ are hermitian matrices that commute so that they can be simultaneously diagonalized by a unitary matrix. It therefore follows that there exists a basis in which $Z^{(1)}$ and $Z^{(2)}$ are simultaneously diagonal; that is, $\lambda_{7}=-\lambda_{6}$. This observation will be crucial to many of the considerations of this section.

The existence of a basis in which $\lambda_{7}=-\lambda_{6}$ provides another opportunity for checking the number of $2 \mathrm{HDM}$ independent parameters. Once this basis is achieved, one can always rephase one of the Higgs fields such that $\lambda_{6}$ and $\lambda_{7}$ are real. This leaves seven real parameters $\left(m_{11}^{2}, m_{22}^{2}, \lambda_{1,2,3,4,6}\right)$ and two complex parameters $\left(m_{12}^{2}\right.$ and $\left.\lambda_{5}\right)$ for a total of eleven independent parameters.

The region of the Higgs potential parameter space where $\lambda_{1}=\lambda_{2}$ and $\lambda_{7}=-\lambda_{6}$ is especially noteworthy. In this case, $Z^{(1)}$ and $Z^{(2)}$ are both proportional to the $2 \times 2$ unit matrix. This must be true for all basis choices. Hence if $\lambda_{1}=\lambda_{2}$ and $\lambda_{7}=-\lambda_{6}$ holds in one basis then it holds in all bases. A (basis-independent) invariant condition can be given for this case. Consider

$$
2 \operatorname{Tr}\left[Z^{(1)}\right]^{2}-\left(\operatorname{Tr} Z^{(1)}\right)^{2}=\left(\lambda_{1}-\lambda_{2}\right)^{2}+4\left|\lambda_{6}+\lambda_{7}\right|^{2}
$$

If this invariant quantity vanishes, then $\lambda_{1}=\lambda_{2}$ and $\lambda_{7}=-\lambda_{6}$.

It is important to emphasize that the latter represents a region of Higgs parameter space. That is, in this region of the parameter space, any change of basis will generally modify the Higgs potential parameters, subject to the condition that $\lambda_{1}=\lambda_{2}$ and $\lambda_{7}=-\lambda_{6}$. Two results will prove useful in the following. First, if $\lambda_{1}=\lambda_{2}$ and $\lambda_{7}=-\lambda_{6}$, then one can always find a basis in which all the $\lambda_{i}$ are real. ${ }^{6}$ Second, if $\lambda_{1}=\lambda_{2}$ and $\lambda_{7}=-\lambda_{6}$, then

\footnotetext{
${ }^{6}$ A simple phase redefinition can render $\lambda_{6}$ and $\lambda_{7}$ real. The nontrivial part of the proof, which is given in ref. 25], demonstrates that a basis always exists in which $\lambda_{5}, \lambda_{6}$ and $\lambda_{7}$ are simultaneously real.
} 
there exists a basis in which $\lambda_{5}$ is real and $\lambda_{6}=\lambda_{7}=0$. The proof of the latter result is simple given the former result. First, transform to a basis in which all the $\lambda_{i}$ are real. Then, make one further $\mathrm{U}(2)$ transformation given by eq. (B2) with $\theta=\pi / 4$ and $\gamma+\zeta=\pi / 2$. It is easy to check that in the final basis, $\lambda_{6}=\lambda_{7}=0$. Finally, since $\lambda_{5}$ is the only potentially complex parameter remaining, it can be rendered real with a phase redefinition of one of the two Higgs fields.

If $Y_{11}=Y_{22}$ and $Y_{12}=0$ then $Y$ is proportional to the $2 \times 2$ unit matrix. Again, one may conclude that if $Y_{11}=Y_{22}$ and $Y_{12}=0$ holds in one basis then it holds in all bases. Combining the two special cases just considered yields an exceptional region of the Higgs potential parameter space in which $Y_{11}=Y_{22}, Y_{12}=0, \lambda_{1}=\lambda_{2}$ and $\lambda_{7}=-\lambda_{6}$. A possible discrete symmetry, which is respected by the scalar Lagrangian and characterizes this exceptional choice of parameters, is:

$$
\Phi_{1} \rightarrow e^{i \psi} \Phi_{2}^{*}, \quad \Phi_{2} \rightarrow-e^{i \psi} \Phi_{1}^{*}
$$

where $\psi$ is an arbitrary phase. ${ }^{7}$ It is straightforward to show that if eq. (37) is a symmetry in one basis, then it is a symmetry in all bases. The results above also imply that within the exceptional region of parameter space, a basis exists in which $Y_{a \bar{b}} \propto \delta_{a \bar{b}}, \lambda_{1}=\lambda_{2}, \lambda_{5}$ is real and $\lambda_{6}=\lambda_{7}=0$.

Alternatively, the exceptional region can be characterized by two simultaneous ordinary $\mathbf{Z}_{2}$ symmetries. In particular, consider a basis in which the scalar Lagrangian respects the two discrete symmetries: (i) $\Phi_{1} \rightarrow \Phi_{1}, \Phi_{2} \rightarrow-\Phi_{2}$ [to be discussed further in section ஹB] and (ii) $\Phi_{1} \rightarrow \Phi_{2}, \Phi_{2} \rightarrow-\Phi_{1}$. Symmetry (i) implies that $Y_{12}=0$ and $\lambda_{6}=\lambda_{7}=0$, while symmetry (ii) implies that $Y_{11}=Y_{22}, Y_{12}^{*}=-Y_{12}, \lambda_{5}^{*}=\lambda_{5}$, and $\lambda_{6}=-\lambda_{7}^{*}$. If symmetries (i) and (ii) are simultaneously satisfied (in the same basis), then $Y_{a \bar{b}} \propto \delta_{a \bar{b}}, \lambda_{1}=\lambda_{2}, \lambda_{5}$ is real and $\lambda_{6}=\lambda_{7}=0$, which indeed corresponds to the exceptional region of parameter space in a particular basis.

\footnotetext{
${ }^{7}$ Since eq. (37) involves the transformation of the scalar fields to their complex conjugates, it follow that this discrete symmetry incorporates a charge conjugation transformation.
} 


\section{B. The discrete symmetry $\Phi_{1} \rightarrow \Phi_{1}, \Phi_{2} \rightarrow-\Phi_{2}$}

We consider first the discrete symmetry $\Phi_{1} \rightarrow+\Phi_{1}, \Phi_{2} \rightarrow-\Phi_{2}$, which is realized for some choice of basis. This discrete symmetry implies that $m_{12}^{2}=\lambda_{6}=\lambda_{7}=0$. It is a discrete $\mathbf{Z}_{2}$ subgroup of the Peccei-Quinn $\mathrm{U}(1)$ symmetry $\Phi_{1} \rightarrow e^{i \alpha} \Phi_{1}, \Phi_{2} \rightarrow e^{-i \alpha} \Phi_{2}{ }^{8}$ The basis-independent conditions for the $\Phi_{1} \rightarrow+\Phi_{1}, \Phi_{2} \rightarrow-\Phi_{2}$ discrete symmetry can be expressed in terms of commutators of matrices constructed from the Higgs potential parameter tensors $Y$ and $Z$. An alternate approach in terms of invariants constructed from $Z$ and the eigenvectors of $Y$ is discussed in Appendix $\mathrm{B}$, along with the explicit eigenvector construction.

Our strategy for deducing the relevant commutator conditions is as follows. We first transform to a basis where $\lambda_{7}=-\lambda_{6}$ [this is always possible as discussed below eq. (35)]. In this basis, we search for commutators that vanish when $Y_{12}=\lambda_{6}=0$. We then conclude that the vanishing of such commutators implies that some basis must exist where $m_{12}^{2}=$ $\lambda_{6}=\lambda_{7}=0$. We shall make use of the following two matrices: ${ }^{9}$

$$
Z_{c \bar{d}}^{(11)} \equiv Z_{b \bar{a}}^{(1)} Z_{a \bar{b} c \bar{d}}, \quad Y_{c \bar{d}}^{(1)} \equiv Y_{b \bar{a}} Z_{a \bar{b} c \bar{d}}
$$

Then, consider the following two commutators evaluated in a basis where $\lambda_{7}=-\lambda_{6}$ :

$$
\begin{aligned}
{\left[Z^{(1)}, Y\right] } & =\left(\lambda_{1}-\lambda_{2}\right)\left(\begin{array}{cc}
0 & Y_{12} \\
-Y_{12}^{*} & 0
\end{array}\right), \\
{\left[Z^{(1)}, Z^{(11)}\right] } & =\left(\lambda_{1}-\lambda_{2}\right)^{2}\left(\begin{array}{cc}
0 & \lambda_{6} \\
-\lambda_{6}^{*} & 0
\end{array}\right),
\end{aligned}
$$

First we assume that $\lambda_{1} \neq \lambda_{2}$ in the $\lambda_{7}=-\lambda_{6}$ basis. In this case, we find that

$$
\left[Z^{(1)}, Y\right]=\left[Z^{(1)}, Z^{(11)}\right]=0
$$

is the basis independent condition that guarantees the existence of a basis in which $m_{12}^{2}=$ $\lambda_{6}=\lambda_{7}=0$. As an example, in Appendix $\mathbf{C}$, we demonstrate that if a $2 \mathrm{HDM}$ scalar potential

\footnotetext{
${ }^{8}$ If $\lambda_{5}=0$ in the same basis where the discrete $\mathbf{Z}_{2}$ symmetry is realized, then the scalar Lagrangian respects the full Peccei-Quinn U(1) global symmetry [2].

${ }^{9}$ Diagrammatically, $Z^{(11)}$ corresponds to a bubble-on-a-bubble with two legs (i.e., a snowman) and $Y^{(1)}$ corresponds to a bubble on two legs with a cross at the top of the bubble.
} 
respects a permutation symmetry $\Phi_{1} \leftrightarrow \Phi_{2}$ in some basis, then eq. (41) is satisfied. This result implies that in some other basis the discrete symmetry $\Phi_{1} \rightarrow \Phi_{1}, \Phi_{2} \rightarrow-\Phi_{2}$ must be manifest.

If $\lambda_{1}=\lambda_{2}$ and $\lambda_{7}=-\lambda_{6}$, then $Z_{a \bar{b}}^{(1)}, Z_{a \bar{b}}^{(11)} \propto \delta_{a \bar{b}}$, and eqs. (139) and (40) automatically vanish. In this case, we are free to transform to a basis in which $Y$ is diagonal. The following commutator is now relevant (for $\lambda_{7}=-\lambda_{6}$ and $Y_{12}=0$ ):

$$
\left[Y^{(1)}, Y\right]=\left(Y_{11}-Y_{22}\right)^{2}\left(\begin{array}{cc}
0 & -\lambda_{6} \\
\lambda_{6}^{*} & 0
\end{array}\right) \text {. }
$$

Assuming that $Y_{11} \neq Y_{22}$, we find that:

$$
\left[Y^{(1)}, Y\right]=0 \quad \text { and } \quad Z_{a \bar{b}}^{(1)} \propto \delta_{a \bar{b}}
$$

are basis independent conditions that guarantee the existence of a basis in which $m_{12}^{2}=$ $\lambda_{6}=\lambda_{7}=0$.

None of the above arguments apply to the exceptional region of parameter space [see section IIIA where $Y_{11}=Y_{22}, Y_{12}=0, \lambda_{1}=\lambda_{2}$ and $\lambda_{7}=-\lambda_{6}$. In this case, all three commutators [eqs. (39), (40) and (42)] automatically vanish since all the matrices involved are proportional to the unit matrix. However, as noted at the end of section 【II in the exceptional region, there exists a basis in which the $\mathbf{Z}_{\mathbf{2}}$ discrete symmetry $\Phi_{1} \rightarrow \Phi_{1}$, $\Phi_{2} \rightarrow-\Phi_{2}$ is manifest. Thus, no further invariant conditions are required.

Finally, in the basis where the discrete symmetry is manifest $\left(m_{12}^{2}=\lambda_{6}=\lambda_{7}=0\right)$, eq. (A7) implies that $\operatorname{Im}\left(\lambda_{5} e^{2 i \xi}\right)=0$. One can always choose $\lambda_{5}$ real (and hence $\xi=0$ ) by redefining the phase of $\Phi_{2}$. We conclude that the Higgs scalar potential of this model is CP-conserving. Further considerations of CP invariance will be given in section $\amalg$ II

We can generalize the results of this section in two ways. First, if there exists a basis in which $\lambda_{6}=\lambda_{7}=0$ but $m_{12}^{2} \neq 0$, then the $\mathbf{Z}_{2}$ discrete symmetry is softly broken [20, 23]. A basis-independent characterization of the softly-broken $\mathbf{Z}_{2}$ discrete symmetry is:

$$
\left[Z^{(1)}, Z^{(11)}\right]=0 \quad \text { and } \quad\left[Z^{(1)}, Y\right] \neq 0
$$

assuming that $\lambda_{1} \neq \lambda_{2}$ in a basis where $\lambda_{7}=-\lambda_{6}$. In the case of $\lambda_{1}=\lambda_{2}$ and $\lambda_{7}=-\lambda_{6}$, we proved in section $\llbracket \mathrm{A}$ that one can always transform to a new basis in which $\lambda_{5}$ is real and $\lambda_{6}=\lambda_{7}=0$. One can then check that the $\mathbf{Z}_{2}$ discrete symmetry is softly-broken if:

$$
\left[Y^{(1)}, Y\right] \neq 0, \quad \text { and } \quad Z_{a \bar{b}}^{(1)} \propto \delta_{a \bar{b}}
$$


Second, if $\lambda_{5}=0$ in a basis where $m_{12}^{2}=\lambda_{6}=\lambda_{7}=0$, then the Higgs potential respects a Peccei-Quinn global U(1) symmetry. That is, $H_{1}$ and $H_{2}$ number are individually conserved. To find invariant conditions that must be satisfied when this symmetry is realized, it is convenient to work in the eigenbasis of $Y .{ }^{10}$ We assume that its eigenvalues are nondegenerate so that they label $H_{1}$ and $H_{2}$. $Z$ will conserve $H_{i}$ number in this basis if every component of $Z$ has equal number of incoming and outgoing $H_{1}$ lines and $H_{2}$ lines. In this case, $Z$ with its incoming lines weighted by their $Y$ eigenvalues will be equal to $Z$ with its outgoing lines weighted by their $Y$ eigenvalues. This difference corresponds to the following "commutator-like" fourth rank tensor:

$$
X_{a \bar{b} c \bar{d}} \equiv Y_{a \bar{e}} Y_{c \bar{f}} Z_{e \bar{b} f \bar{d}}-Y_{e \bar{b}} Y_{f \bar{d}} Z_{a \bar{e} c \bar{f}}
$$

We can see that $X_{a \bar{b} c \bar{d}}=0$ corresponds to the Peccei-Quinn symmetry as follows. Hermiticity properties of $Y$ and $Z$ imply that:

$$
X_{a \bar{b} c \bar{d}}=-\left(X_{b \bar{a} d \bar{c}}\right)^{*}
$$

Suppose that $\lambda_{1} \neq \lambda_{2}$ in a basis where $\lambda_{7}=-\lambda_{6}$. If $\left[Z^{(1)}, Y\right]=0$ then $Y_{12}=0$. If $\lambda_{1}=\lambda_{2}$ and $\lambda_{7}=-\lambda_{6}$ then one can transform to a new basis in which $Y_{12}=0$. In either case, with $\lambda_{7}=-\lambda_{6}$ and $Y_{12}=0$, we find

$$
X_{a \bar{b} c \bar{d}} \equiv\left(Y_{11}-Y_{22}\right) x_{a \bar{b} c \bar{d}}
$$

where

$$
\begin{array}{ll}
x_{1111}=0, & x_{2222}=0, \\
x_{1122}=x_{2211}=0, & x_{1221}=x_{2112}=0, \\
x_{1212}=\left(Y_{11}+Y_{22}\right) \lambda_{5}, & x_{2121}=-\left(Y_{11}+Y_{22}\right) \lambda_{5}^{*}, \\
x_{1112}=x_{1211}=Y_{11} \lambda_{6}, & x_{1121}=x_{2111}=-Y_{11} \lambda_{6}^{*}, \\
x_{2212}=x_{1222}=Y_{22} \lambda_{7}, & x_{2221}=x_{2122}=-Y_{22} \lambda_{7}^{*} .
\end{array}
$$

${ }^{10}$ If the eigenvalues of $Y$ are degenerate, then one can work in in the eigenbasis of $Z^{(1)}$, assuming that its eigenvalues are non-degenerate. In this case, the arguments above still apply with the obvious modifications. These considerations are not applicable in the case where both $Y$ and $Z^{(1)}$ have degenerate eigenvalues, which corresponds to the exceptional region of the Higgs potential parameter space [as defined in section 
Thus, if $Y_{11} \neq Y_{22}$, then the additional conditions $X_{a \bar{b} c \bar{d}}=0$ guarantee that a basis exists in which $m_{12}^{2}=\lambda_{5}=\lambda_{6}=\lambda_{7}=0$.

If $Y_{a \bar{b}} \propto \delta_{a \bar{b}}$, then $X_{a \bar{b}} c \bar{d}=0$ automatically, and we consider instead:

$$
\widetilde{X}_{a \bar{b} c \bar{d}} \equiv Z_{a \bar{e}}^{(1)} Z_{c \bar{f}}^{(1)} Z_{e \bar{b} f \bar{d}}-Z_{e \bar{c}}^{(1)} Z_{f \bar{d}}^{(1)} Z_{a \bar{e} c \bar{f}}
$$

The analysis is nearly identical as for $X$ above with the replacements $Y_{11} \rightarrow \lambda_{1}+\lambda_{4}$ and

$Y_{22} \rightarrow \lambda_{2}+\lambda_{4}$. Assuming $\lambda_{1} \neq \lambda_{2}$ in a basis where $\lambda_{7} \neq-\lambda_{6}$, the conditions $\widetilde{X}_{a \bar{b} c \bar{d}}=0$ guarantee that a basis exists in which $m_{12}^{2}=\lambda_{5}=\lambda_{6}=\lambda_{7}=0$. Finally, at the exceptional point of parameter space where $Y_{11}=Y_{22}, Y_{12}=0, \lambda_{1}=\lambda_{2}$ and $\lambda_{7}=-\lambda_{6}$, we know it is possible to transform to a basis where $\lambda_{6}=\lambda_{7}=0$. However, in general $\lambda_{5} \neq 0$, and we have found no additional covariant condition that requires $\lambda_{5}=0$ in the same basis.

\section{Explicit and spontaneous CP-violation}

The full power of the U(2)-invariants emerges when one studies CP-violating theories. Thus, the question naturally arises: what are the invariant conditions that determine whether the theory is $\mathrm{CP}$-conserving or CP-violating. These conditions are the analogs of the Jarlskog invariant conditions for CP-violation [16] in the mixing of the quark generations. We first consider conditions such that the Higgs potential explicitly conserves CP, independently of the value of the vacuum expectation value. In the generic basis, the Higgs potential preserves the $\mathrm{CP}$ symmetry if there exists a $\mathrm{U}(2)$ transformation such that the resulting transformed parameters of the Higgs scalar potential are real, i.e., $Y_{a \bar{b}}=\left(Y_{a \bar{b}}\right)^{*}$ and $Z_{a \bar{b} c \bar{d}}=\left(Z_{a \bar{b} c \bar{d}}\right)^{*}$. Using eqs. (3) and (44), these conditions are equivalent to: $Y_{a \bar{b}}=Y_{b \bar{a}}$ and $Z_{a \bar{b} c \bar{d}}=Z_{b \bar{a} d \bar{c}}$. However, it may be difficult in general to find the basis where all the Higgs potential parameters are real or prove that such a basis does not exist. An alternative strategy is to find $\mathrm{U}(2)$-invariant quantities constructed from $Y_{a \bar{b}}$ and $Z_{a \bar{b} c \bar{d}}$ that are complex in a CP-violating theory. In an explicitly CP-conserving theory, the existence of the real basis implies that any such invariant is real. Hence, the non-vanishing of the imaginary part of a potentially complex invariant would provide a test for explicit CP-violation.

We anticipate two invariants - one involving only factors of $Z$ tensors and one involving $Y$ and $Z$. To see this, we first transform to a basis where $\lambda_{6}=-\lambda_{7}$ [see section ஹA]. The relative phase between $\Phi_{1}$ and $\Phi_{2}$ can be chosen such that $\lambda_{6}, \lambda_{7}$ are both real. Thus, 


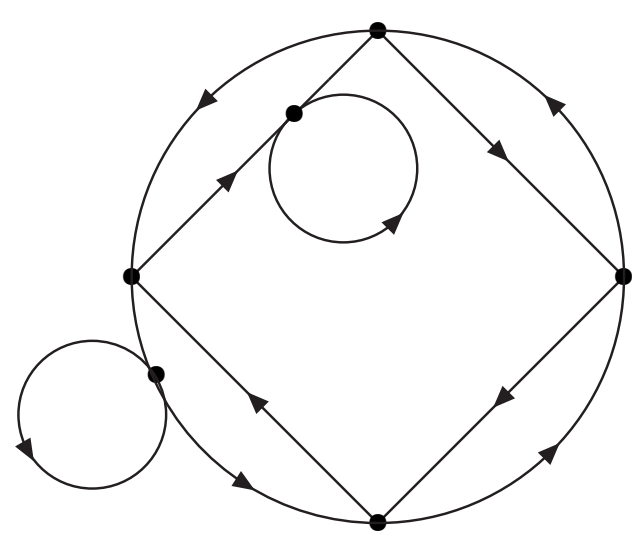

(a)

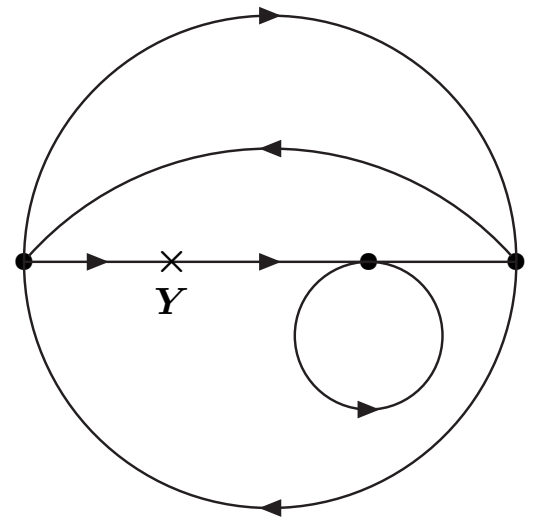

(b)

FIG. 3: Diagrams corresponding to the potentially complex invariants of eq. (151).

assuming that $\lambda_{6} \neq 0$, there remain two independent phases: $\arg \left(m_{12}^{2}\right)$ and $\arg \left(\lambda_{5}\right)$. We have discovered two potentially complex invariants:

$$
Z_{a \bar{b} c \bar{d}} Z_{b \bar{f}}^{(2)} Z_{d \bar{h}}^{(2)} Z_{f \bar{a} j \bar{k}} Z_{k \bar{j} m \bar{n}} Z_{n \bar{m} h \bar{c}}, \quad Z_{a \bar{b} c \bar{d}} Y_{d \bar{e}} Z_{e \bar{g}}^{(2)} Z_{g \bar{c} b \bar{a}}
$$

Using the diagrammatic rules introduced in section [1] [see Fig. 1], the invariants of eq. (51) are depicted in Fig. 3. Applying the CP transformation to the above diagrams changes the direction of the lines. Hence, an invariant can be complex if its diagram looks different when the arrows are reversed. This requirement is satisfied for the above diagrams. As a result, one can easily identify manifestly real invariants, but there is no guarantee that an invariant corresponding to a CP-asymmetric diagram is complex.

Although we have succeeded in finding two potentially complex invariants, it does not necessarily follow that the Higgs potential is CP-invariant if the two invariants of eq. (51) are real. The necessary and sufficient conditions for an explicitly CP-invariant 2HDM potential (in terms of $\mathrm{U}(2)$-invariants) have been obtained in ref. [25], where it is shown that in the $\lambda_{7}=-\lambda_{6}$ basis, both invariants of eq. (51) vanish if either $\lambda_{6}=0$ or if $\lambda_{1}=\lambda_{2}$. In each of these two cases, one must seek out a new invariant. In ref. [25], the two new invariants are found, and a proof is given showing that the reality of all four (potentially complex) invariants is both necessary and sufficient for an explicitly CP-conserving Higgs potential.

If the Higgs potential is explicitly CP-conserving, it is still possible that the Higgs vacuum does not respect the CP symmetry. This is the case of spontaneously broken CP invariance [1]. In particular, the four potentially complex invariants noted above are real, 
and the so-called "real basis" exists in which all the Higgs potential parameters are real. In this basis the Higgs vacuum expectation value has a phase determined by the real Higgs potential parameters [see eq. (A7)]. The existence of this complex phase is not necessarily a signal for spontaneous CP-violation. One must prove that this phase cannot be removed by a further change of basis (subject to the condition that the Higgs potential parameters remain real). Appendix C]provides an example (first discussed in ref. [26]) of a CP-conserving $2 \mathrm{HDM}$ in which the complex phase of the vacuum expectation value appears in the "real basis." In ref. [25], the explicit transformation between the two real bases that removes the complex phase is exhibited.

Thus, it would be useful to find a set of invariant conditions to establish the existence or non-existence of spontaneous CP-violation. Having first proved that the Higgs potential explicitly conserves CP (using the invariant conditions of ref. [25]), one can prove or rule out the existence of spontaneous CP-violation by employing three invariants first obtained in ref. [19]. ${ }^{11}$ We prove below that the Higgs sector ${ }^{12}$ conserves CP if and only if $I_{1}, I_{2}$ and $I_{3}$ are real, where

$$
\begin{aligned}
& -\frac{1}{2} v^{2} I_{1} \equiv \operatorname{Tr}\left(V Y Z^{(1)}\right) \\
& \frac{1}{4} v^{4} I_{2} \equiv(V Y)_{b \bar{a}}(V Y)_{d \bar{c}} Z_{a \bar{b} c \bar{d}} \\
& \frac{1}{4} v^{2} I_{3} \equiv\left(V Z^{(1)}\right)_{b \bar{a}} V_{d \bar{c}}\left[\frac{1}{4} v^{2} Z_{c \bar{e}}^{(1)} Z_{a \bar{b} e \bar{d}}+Y_{a \bar{d}} Z_{c \bar{b}}^{(1)}\right] \text {. }
\end{aligned}
$$

The factors of $v^{2}$ have been introduced in eqs. (152)-(154) so that the $I_{i}$ are dimensionless. Note that $I_{1}, I_{2}$ and $I_{3}$ are invariants with respect to the full $\mathrm{U}(2)$ Higgs flavor symmetry.

We may employ the scalar potential minimum conditions [eq. (8)] to eliminate $Y$ in the expressions for $I_{1}, I_{2}$ and $I_{3}$ :

$$
\begin{aligned}
I_{1} & \equiv\left(Z^{(1)} V\right)_{b \bar{a}} V_{d \bar{c}} Z_{a \bar{b} c \bar{d}} \\
I_{2} & \equiv V_{a \bar{b}} V_{d \bar{c}} V_{h \bar{g}} V_{r \bar{p}} Z_{b \bar{e} g \bar{h}} Z_{c \bar{f} p \bar{r}} Z_{e \bar{a} f \bar{d}} \\
I_{3} & \equiv\left(V Z^{(1)}\right)_{b \bar{a}} V_{d \bar{c}}\left[Z_{c \bar{e}}^{(1)} Z_{a \bar{b} e \bar{d}}-2 Z_{c \bar{d}}^{(1)} V_{f \bar{e}} Z_{a \bar{b} e \bar{f}}\right]
\end{aligned}
$$

${ }^{11}$ We prefer to express the CP-invariants in terms of the $Y_{a \bar{b}}, Z_{a \bar{b}} c \bar{d}$ and $v_{a}$, following the work of ref. [15]. However, only two of the three invariants were explicitly given in this reference.

${ }^{12}$ In this context, the Higgs sector includes the Higgs Lagrangian and its coupling to the gauge bosons via the covariant derivative in the Higgs kinetic energy term. If the Higgs-fermion Yukawa interactions are also included, then additional CP-odd invariants based on invariants that involve both Higgs potential parameters and the Higgs-fermion Yukawa coupling matrices must be considered [15]. 
The invariants above are most easily evaluated in the Higgs basis [eq. (27)]. Using eq. (B10), we end up with:

$$
\operatorname{Im} I_{1}=\operatorname{Im}\left[Z_{6} Z_{7}^{*}\right], \quad \operatorname{Im} I_{2}=\operatorname{Im}\left[Z_{5}^{*} Z_{6}^{2}\right], \quad \operatorname{Im} I_{3}=\operatorname{Im}\left[Z_{5}^{*}\left(Z_{6}+Z_{7}\right)^{2}\right]
$$

The necessary and sufficient conditions for a CP-invariant scalar Higgs potential and a CP-conserving Higgs vacuum are: $\operatorname{Im} I_{1}=\operatorname{Im} I_{2}=\operatorname{Im} I_{3}=0$. In fact, there is at most two independent relative phases among $I_{1}, I_{2}$ and $I_{3}$. However, there are cases where two of the three invariants are real and only one has an imaginary part: ${ }^{13}$

$$
\begin{aligned}
& \operatorname{Im} I_{1}=\operatorname{Im} I_{2}=0 \quad \text { if } \quad Z_{6}=0, \\
& \operatorname{Im} I_{1}=\operatorname{Im} I_{3}=0 \quad \text { if } \quad Z_{7}=-Z_{6} \text {, } \\
& \operatorname{Im} I_{2}=\operatorname{Im} I_{3}=0 \text { if } Z_{5}=0 \text {, }
\end{aligned}
$$

which shows that one must check all three invariants before determining whether the Higgs sector is CP-invariant. ${ }^{14}$

If $I_{1}, I_{2}$ and $I_{3}$ are real one can always perform a phase rotation $H_{2} \rightarrow e^{i \psi} H_{2}$ such that the resulting Higgs basis couplings are all real for some choice of $\psi$. [Note that the Higgs basis squared mass parameter, $M_{12}^{2}$ is then automatically real by virtue of eq. (A25).] The Higgs sector is then manifestly CP-conserving. Conversely, if the Higgs sector is CP-conserving, then some basis must exist in which the Higgs potential parameters and the Higgs field vacuum expectation values are simultaneously real. Thus, the invariant quantities $I_{1}, I_{2}$ and $I_{3}$ must be real.

\section{CP-INVARIANT 2HDM BOSONIC COUPLINGS IN TERMS OF INVARI- ANT PARAMETERS}

The phenomenology of the two-Higgs doublet model depends in detail on the various couplings of the Higgs bosons to gauge bosons, Higgs bosons and fermions [4]. We assume that the Higgs sector is CP-conserving, so we can work in a basis in which the vacuum expectation values are both real and non-negative, and all the parameters of the scalar

${ }^{13}$ If $Z_{7}=0$, then $\operatorname{Im} I_{1}=\operatorname{Im}\left(I_{2}-I_{3}\right)=0$.

${ }^{14}$ If $Y_{a \bar{b}}=0$ then $I_{1}=I_{2}=0$. More generally, if $Y_{a \bar{b}} \propto \delta_{a \bar{b}}$, then $Y_{3}=0$. Consequently, eq. (31) implies that $Z_{6}=0$, and $I_{3}$ is the only potentially complex invariant. 
potential are real. In this case, it is sufficient to consider $\mathrm{SO}(2)$ rotations among different basis choices; hence, there is no need to distinguish unbarred and barred indices, and we drop the bars in what follows. Moreover, within this set of "real" basis choices, the Higgs basis is unique. Thus, eq. (B10) implies that the $\mathrm{SO}(2)$-invariants, $Y_{i}$ and $Z_{i}$, coincide with the Higgs potential parameters in the Higgs basis $\left(M_{11}^{2}, M_{22}^{2}, M_{12}^{2}\right.$ and the $\Lambda_{i}$, introduced in Appendix A].

Since CP is conserved by assumption, there is no mixing between the CP-even Higgs bosons, $h$ and $H$ and the CP-odd Higgs boson, $A$. In an arbitrary real basis, we define the angle $\alpha$ such that

$$
\begin{aligned}
& h=-\left(\sqrt{2} \operatorname{Re} \Phi_{1}^{0}-v_{1}\right) s_{\alpha}+\left(\sqrt{2} \operatorname{Re} \Phi_{2}^{0}-v_{2}\right) c_{\alpha}, \\
& H=\left(\sqrt{2} \operatorname{Re} \Phi_{1}^{0}-v_{1}\right) c_{\alpha}+\left(\sqrt{2} \operatorname{Re} \Phi_{2}^{0}-v_{2}\right) s_{\alpha} .
\end{aligned}
$$

For a basis-independent description, we define the unit vector:

$$
\widehat{n}=\left(\begin{array}{c}
c_{\alpha} \\
s_{\alpha}
\end{array}\right)
$$

Then, the two basis-independent quantities associated with the CP-even mass eigenstates are:

$$
\widehat{n}_{a} \widehat{v}_{a}=\cos (\beta-\alpha) \equiv c_{\beta-\alpha}, \quad \epsilon_{a b} \widehat{n}_{a} \widehat{v}_{b}=\sin (\beta-\alpha) \equiv s_{\beta-\alpha} .
$$

Thus, the angle $\beta-\alpha$ represents the direction of the CP-even Higgs mass eigenstates relative to the Higgs basis. That is,

$$
\begin{aligned}
& h=\varphi_{1}^{0} \sin (\beta-\alpha)+\varphi_{2}^{0} \cos (\beta-\alpha), \\
& H=\varphi_{1}^{0} \cos (\beta-\alpha)-\varphi_{2}^{0} \sin (\beta-\alpha),
\end{aligned}
$$

where $\varphi_{1}^{0}$ and $\varphi_{2}^{0}$ are defined in eq. (A11). Henceforth, we will continue to use the notation $s_{\beta-\alpha}$ and $c_{\beta-\alpha}$ for the basis-independent quantities [eq. (64)] despite the fact that $\beta$ and $\alpha$ are separately basis-dependent.

In the Higgs basis, the CP-even squared mass matrix takes on a rather simple form:

$$
\mathcal{M}^{2}=\left(\begin{array}{cc}
\Lambda_{1} v^{2} & \Lambda_{6} v^{2} \\
\Lambda_{6} v^{2} & m_{A}^{2}+\Lambda_{5} v^{2}
\end{array}\right)
$$

where

$$
m_{A}^{2}=m_{H^{ \pm}}^{2}-\frac{1}{2} v^{2}\left(\Lambda_{5}-\Lambda_{4}\right)
$$


and $m_{H^{ \pm}}^{2}$ is given by eq. (A26). After diagonalization of $\mathcal{M}^{2}$, one determines the CP-even Higgs squared-masses:

$$
m_{H, h}^{2}=\frac{1}{2}\left[m_{A}^{2}+v^{2}\left(\Lambda_{1}+\Lambda_{5}\right) \pm \sqrt{\left[m_{A}^{2}+\left(\Lambda_{5}-\Lambda_{1}\right) v^{2}\right]^{2}+4 \Lambda_{6}^{2} v^{4}}\right]
$$

and the angle $\beta-\alpha$ can be determined from the following results:

$$
\begin{aligned}
\tan [2(\beta-\alpha)] & =\frac{2 \Lambda_{6} v^{2}}{m_{A}^{2}+\left(\Lambda_{5}-\Lambda_{1}\right) v^{2}}, \\
\sin [2(\beta-\alpha)] & =\frac{-2 \Lambda_{6} v^{2}}{m_{H}^{2}-m_{h}^{2}} .
\end{aligned}
$$

From these expressions one can derive numerous relations among the squared-masses, invariant coupling parameters $\Lambda_{1}, \Lambda_{5}$ and $\Lambda_{6}$, and $\beta-\alpha$ [21, 24].

The Higgs couplings to gauge bosons follow from gauge invariance. The properties of the three-point and four-point Higgs boson-vector boson couplings are conveniently summarized by listing the couplings that are proportional to either $\sin (\beta-\alpha)$ or $\cos (\beta-\alpha)$, and the couplings that are independent of $\beta-\alpha[4]$ :

\begin{tabular}{lll}
$\frac{\cos (\beta-\alpha)}{H}$ & $\frac{\sin (\beta-\alpha)}{h W^{+} W^{-}}$ & $\underline{\text { angle-independent }}$ \\
$H Z Z$ & $h Z Z$ & - \\
$Z A h$ & $Z A H$ & $Z H^{+} H^{-}, \gamma H^{+} H^{-}$ \\
$W^{ \pm} H^{\mp} h$ & $W^{ \pm} H^{\mp} H$ & $W^{ \pm} H^{\mp} A$ \\
$Z W^{ \pm} H^{\mp} h$ & $Z W^{ \pm} H^{\mp} H$ & $Z W^{ \pm} H^{\mp} A$ \\
$\gamma W^{ \pm} H^{\mp} h$ & $\gamma W^{ \pm} H^{\mp} H$ & $\gamma W^{ \pm} H^{\mp} A$ \\
\multicolumn{1}{c}{-} & - & $V V \phi \phi, V V A A, V V H^{+} H^{-}$
\end{tabular}

where $\phi=h$ or $H$ and $V V=W^{+} W^{-}, Z Z, Z \gamma$ or $\gamma \gamma$. Indeed, the Higgs boson-vector boson couplings are basis-independent.

The three-point and four-point Higgs self-couplings are more complicated. Nevertheless, it is clear that one can express these couplings in terms of $s_{\beta-\alpha}, c_{\beta-\alpha}$ and the invariant coupling parameters, $\Lambda_{i}$. The simplest way to obtain the Higgs self-couplings is to work in the Higgs basis, using eqs. (A11), (65) and (66). For example,

$$
\begin{aligned}
g_{h h h} & =-3 v\left[\Lambda_{1} s_{\beta-\alpha}^{3}+\Lambda_{345} s_{\beta-\alpha} c_{\beta-\alpha}^{2}+3 \Lambda_{6} c_{\beta-\alpha} s_{\beta-\alpha}^{2}+\Lambda_{7} c_{\beta-\alpha}^{3}\right], \\
g_{h h h h} & =-3\left[\Lambda_{1} s_{\beta-\alpha}^{4}+\Lambda_{2} c_{\beta-\alpha}^{4}+2 \Lambda_{345} c_{\beta-\alpha}^{2} s_{\beta-\alpha}^{2}+4 \Lambda_{6} c_{\beta-\alpha} s_{\beta-\alpha}^{3}+4 \Lambda_{7} c_{\beta-\alpha}^{3} s_{\beta-\alpha}\right],
\end{aligned}
$$


where $\Lambda_{345} \equiv \Lambda_{3}+\Lambda_{4}+\Lambda_{5}$. A complete list of the trilinear and quartic Higgs couplings in terms of the invariant coupling parameters and $\beta-\alpha$ can be found in ref. [24]. ${ }^{15}$

One can generalize the analysis of this section by relaxing the requirement that the Higgs vacuum expectation values are positive. That is, we consider the set of all basis choices related by $\mathrm{O}(2)$ rotations. In this case, two possible Higgs bases exist, since one may perform the transformation $H_{1} \rightarrow H_{1}, H_{2} \rightarrow-H_{2}$. Under this transformation, $M_{12}^{2}, \Lambda_{6}, \Lambda_{7}$ and $\beta-\alpha$ all change sign (whereas all other Higgs potential parameters are left unchanged). This is not surprising since all the $\mathrm{SO}(2)$-invariants that change sign involve an odd number of $\epsilon_{a b}$ symbols in their definitions. We shall use the term pseudo-invariant to refer to an $\mathrm{SO}(2)$-invariant that changes sign under an $\mathrm{O}(2)$ transformation with determinant equal to -1 . In addition, we note that eqs. (62) and (A10) imply that the physical Higgs fields are related to the Higgs basis fields by:

$$
\begin{aligned}
h & =\left(\sqrt{2} \operatorname{Re} H_{1}^{0}-v\right) s_{\beta-\alpha}+\sqrt{2} \operatorname{Re} H_{2}^{0} c_{\beta-\alpha}, \\
H & =\left(\sqrt{2} \operatorname{Re} H_{1}^{0}-v\right) c_{\beta-\alpha}-\sqrt{2} \operatorname{Re} H_{2}^{0} s_{\beta-\alpha},
\end{aligned}
$$

$H^{ \pm}=H_{2}^{ \pm}$and $A=\sqrt{2} \operatorname{Im} H_{2}^{0}$. Thus, under $H_{1} \rightarrow H_{1}, H_{2} \rightarrow-H_{2}$, the fields $h, H^{ \pm}$and $A$ change sign and $H$ is unchanged. One can now check that eqs. (68)-(174) are indeed invariant with respect to $\mathrm{O}(2)$ transformations, so that physical results do not depend on the choice of basis.

\section{YUKAWA COUPLINGS OF THE 2HDM IN TERMS OF INVARIANT PA- RAMETERS}

The Higgs couplings to fermions are model dependent. The most general structure for the Higgs-fermion Yukawa couplings, often referred to as the type-III model [13], is given in the generic basis by:

$$
-\mathcal{L}_{\mathrm{Y}}=\bar{Q}_{L}^{0} \widetilde{\Phi}_{1} \eta_{1}^{U, 0} U_{R}^{0}+\bar{Q}_{L}^{0} \Phi_{1} \eta_{1}^{D, 0} D_{R}^{0}+\bar{Q}_{L}^{0} \widetilde{\Phi}_{2} \eta_{2}^{U, 0} U_{R}^{0}+\bar{Q}_{L}^{0} \Phi_{2} \eta_{2}^{D, 0} D_{R}^{0}+\text { h.c. }
$$

where $\Phi_{1,2}$ are the Higgs doublets, $\widetilde{\Phi}_{i} \equiv i \sigma_{2} \Phi_{i}^{*}, Q_{L}^{0}$ is the weak isospin quark doublet, and $U_{R}^{0}, D_{R}^{0}$ are weak isospin quark singlets. [The right and left-handed fermion fields are defined

$\overline{15}$ To make contact with the notation of ref. [24], we define: $\lambda \equiv \Lambda_{1}, \lambda_{V} \equiv \Lambda_{2}, \lambda_{T} \equiv \Lambda_{3}+\Lambda_{4}-\Lambda_{5}$, $\lambda_{F} \equiv \Lambda_{5}-\Lambda_{4}, \lambda_{A} \equiv \Lambda_{1}-\Lambda_{5}, \widehat{\lambda} \equiv-\Lambda_{6}$ and $\lambda_{U} \equiv-\Lambda_{7}$. 
as usual: $\psi_{R, L} \equiv P_{R, L} \psi$, where $P_{R, L} \equiv \frac{1}{2}\left(1 \pm \gamma_{5}\right)$.] Here, $Q_{L}^{0}, U_{R}^{0}, D_{R}^{0}$ denote the interaction basis states, which are vectors in the quark flavor space, and $\eta_{1}^{U, 0}, \eta_{2}^{U, 0}, \eta_{1}^{D, 0}, \eta_{2}^{D, 0}$ are matrices in quark flavor space. Clearly, these four matrices are basis-dependent quantities. We have omitted the leptonic couplings in eq. (76); these follow the same pattern as the down-type quark couplings.

In some models, not all the terms in eq. (176) are present at tree-level [1]]. In a type-I model, there exist a basis where $\eta_{2}^{U, 0}=\eta_{2}^{D, 0}=0 .{ }^{16}$ In a type-II model, $\eta_{1}^{U, 0}=\eta_{2}^{D, 0}=0$. The vanishing of certain Higgs-fermion couplings at tree-level can be enforced by a discrete symmetry. For example, if Lagrangian is invariant under $\Phi_{1} \rightarrow+\Phi_{1}, \Phi_{2} \rightarrow-\Phi_{2}$ in some basis, then by demanding additional discrete symmetries of a similar type for the quark fields, one can preserve either the type-I or type-II Higgs-fermion couplings (depending on the precise choice of discrete symmetries) while eliminating the other possible terms in eq. (176). Another well-known example is the MSSM Higgs sector, which exhibits a type-II Higgs-fermion coupling pattern that is enforced by supersymmetry.

For type-I and type-II Higgs-fermion couplings, there is a natural basis choice that is imposed, either by the discrete symmetry or supersymmetry. In this case, $\tan \beta$ becomes a meaningful parameter. However, in the more general type-III model, there is no distinguished basis, and once again $\tan \beta$ is meaningless. To demonstrate this fact more explicitly, we now proceed to write out the type-III Higgs-quark interactions in a basis independent manner. Our strategy is to rewrite the Higgs-fermion interaction [eq. (76)] in the Higgs basis and identify the quark masses. As in section IV we assume that the Higgs potential is CP-conserving so that we can work in a class of bases related by $\mathrm{SO}(2)$ transformations. Thus, the basis-independent quantities considered in this section are invariant with respect to $\mathrm{SO}(2) .{ }^{17}$ However, we shall allow the Higgs-fermion couplings to be complex (and hence

${ }^{16}$ A type-I model can also be defined as a model in which $\eta_{1}^{U, 0}=\eta_{1}^{D, 0}=0$ in some basis. Clearly, the two definitions are equivalent, since the difference in the two conditions is simply an interchange of $\Phi_{1}$ and $\Phi_{2}$ which can be viewed as a change of basis.

17 As noted at the end of section IV one can extend the class of basis transformations to $\mathrm{O}(2)$. In this case, one should distinguish between true $\mathrm{O}(2)$-invariants and pseudo-invariants that change sign under an $\mathrm{O}(2)$ transformation with determinant -1 . In particular, $\beta-\alpha$ and $\rho^{Q}$ [eq. 884] are pseudo-invariants. However as previously noted, the physical Higgs fields $H^{ \pm}, A$ and $h$ all change sign under the transformation $H_{1} \rightarrow H_{1}, H_{2} \rightarrow-H_{2}$ of Higgs basis fields. Therefore, the physical Higgs-fermion couplings given in eq. (87) are invariant with respect to the full $\mathrm{O}(2)$ group of basis transformations. 
CP-violating). Using eq. (A10),

$$
-\mathcal{L}_{Y}=\bar{Q}_{L}^{0} \widetilde{H}_{1} \kappa^{U, 0} U_{R}^{0}+\bar{Q}_{L}^{0} H_{1} \kappa^{D, 0} D_{R}^{0}+\bar{Q}_{L}^{0} \widetilde{H}_{2} \rho^{U, 0} U_{R}^{0}+\bar{Q}_{L}^{0} H_{2} \rho^{D, 0} D_{R}^{0}+\text { h.c. }
$$

where $\widetilde{H}_{i} \equiv i \sigma_{2} H_{i}^{*}$ and

$$
\begin{aligned}
\kappa^{Q, 0} & \equiv \eta_{1}^{Q, 0} c_{\beta}+\eta_{2}^{Q, 0} s_{\beta}, \\
\rho^{Q, 0} & \equiv-\eta_{1}^{Q, 0} s_{\beta}+\eta_{2}^{Q, 0} c_{\beta},
\end{aligned}
$$

for $Q=U$ or $D$. It is easy to see that $\kappa^{Q, 0}$ and $\rho^{Q, 0}$ are basis-independent quantities. First, we introduce the vector (in Higgs flavor space) of matrix quantities: $\eta^{Q, 0} \equiv\left(\eta_{1}^{Q, 0}, \eta_{2}^{Q, 0}\right)$. Then, eqs. (78) and (79) are equivalent to:

$$
\kappa^{Q, 0} \equiv \widehat{v} \cdot \eta^{Q, 0}, \quad \rho^{Q, 0} \equiv \widehat{w} \cdot \eta^{Q, 0}
$$

where the dot products have the usual meaning: $\widehat{v} \cdot \eta^{Q, 0} \equiv \widehat{v}_{a} \eta_{a}^{Q, 0}$ and $\widehat{w} \cdot \eta^{Q, 0} \equiv \epsilon_{a b} \widehat{v}_{a} \eta_{b}^{Q, 0}$. Clearly, $\kappa^{Q, 0}$ and $\rho^{Q, 0}$ are $\mathrm{SO}(2)$-invariant quantities.

The fermion mass eigenstates are related to the interaction eigenstates by biunitary transformations:

$$
\begin{array}{ll}
P_{L} U=V_{L}^{U} P_{L} U^{0}, & P_{R} U=V_{R}^{U} P_{R} U^{0}, \\
P_{L} D=V_{L}^{D} P_{L} D^{0}, & P_{R} D=V_{R}^{D} P_{R} D^{0},
\end{array}
$$

and the Cabibbo-Kobayashi-Maskawa matrix is defined as $K \equiv V_{L}^{U} V_{L}^{D \dagger}$. It is also convenient to define "rotated" coupling matrices:

$$
\begin{array}{rlrl}
\eta_{i}^{U} \equiv V_{L}^{U} \eta_{i}^{U, 0} V_{R}^{U \dagger}, & \kappa^{U} \equiv V_{L}^{U} \kappa^{U, 0} V_{R}^{U \dagger}, & & \rho^{U} \equiv V_{L}^{U} \rho^{U, 0} V_{R}^{U \dagger}, \\
\eta_{i}^{D} \equiv V_{L}^{D} \eta_{i}^{D, 0} V_{R}^{D \dagger}, & \kappa^{D} \equiv V_{L}^{D} \kappa^{D, 0} V_{R}^{D \dagger}, & \rho^{D} \equiv V_{L}^{D} \rho^{D, 0} V_{R}^{D \dagger} .
\end{array}
$$

Note that $\kappa^{Q}$ and $\rho^{Q}(Q=U, D)$ are also invariants, since if we define $\eta^{Q} \equiv\left(\eta_{1}^{Q}, \eta_{2}^{Q}\right)$, then

$$
\kappa^{Q} \equiv \widehat{v} \cdot \eta^{Q}, \quad \rho^{Q} \equiv \widehat{w} \cdot \eta^{Q}
$$

The quark mass terms are identified by replacing the scalar fields with their vacuum expectation values. The unitary matrices $V_{L}^{U}, V_{L}^{D}, V_{R}^{U}$ and $V_{R}^{D}$ are then chosen so that $\kappa^{D}$ and $\kappa^{U}$ are diagonal with real non-negative entries. The resulting quark mass matrices are then diagonal:

$$
M_{D}=\frac{v}{\sqrt{2}} \kappa^{D}, \quad M_{U}=\frac{v}{\sqrt{2}} \kappa^{U} .
$$


In this analysis, we have assumed that the Higgs potential is CP-conserving, in which case we may take $\widehat{v}=(\cos \beta, \sin \beta)$. By construction, the $\kappa^{Q}$ are real diagonal matrices. Eq. (84) then implies that

$$
\operatorname{Im} \eta_{1}^{Q}=-\tan \beta \operatorname{Im} \eta_{2}^{Q}
$$

and $\operatorname{Im} \rho^{Q}=\operatorname{Im} \eta_{2}^{Q} / \cos \beta$. Similarly, $\rho^{Q}$ is typically non-diagonal. Thus, in the most general case, $\rho^{D}$ and $\rho^{U}$ are independent complex non-diagonal matrices.

Finally, from eq. (77), we obtain the Higgs-quark couplings after making use of eqs. (A11), (65) and (66). The end result is:

$$
\begin{aligned}
-\mathcal{L}_{Y}= & \frac{1}{v} \bar{D}\left[M_{D} s_{\beta-\alpha}+\frac{v}{\sqrt{2}}\left(\rho^{D} P_{R}+\rho^{D^{\dagger}} P_{L}\right) c_{\beta-\alpha}\right] D h+\frac{i}{v} \bar{D} M_{D} \gamma_{5} D G^{0} \\
& +\frac{1}{v} \bar{D}\left[M_{D} c_{\beta-\alpha}-\frac{v}{\sqrt{2}}\left(\rho^{D} P_{R}+\rho^{D^{\dagger}} P_{L}\right) s_{\beta-\alpha}\right] D H+\frac{i}{\sqrt{2}} \bar{D}\left(\rho^{D} P_{R}-\rho^{D^{\dagger}} P_{L}\right) D A \\
& +\frac{1}{v} \bar{U}\left[M_{U} s_{\beta-\alpha}+\frac{v}{\sqrt{2}}\left(\rho^{U} P_{R}+\rho^{U^{\dagger}} P_{L}\right) c_{\beta-\alpha}\right] U h-\frac{i}{v} \bar{U} M_{D} \gamma_{5} U G^{0} \\
& +\frac{1}{v} \bar{U}\left[M_{U} c_{\beta-\alpha}-\frac{v}{\sqrt{2}}\left(\rho^{U} P_{R}+\rho^{U^{\dagger}} P_{L}\right) s_{\beta-\alpha}\right] U H-\frac{i}{\sqrt{2}} \bar{U}\left(\rho^{U} P_{R}-\rho^{U^{\dagger}} P_{L}\right) U A \\
& +\left\{\bar{U}\left[K \rho^{D} P_{R}-\rho^{U^{\dagger}} K P_{L}\right] D H^{+}+\frac{\sqrt{2}}{v} \bar{U}\left[K M_{D} P_{R}-M_{U} K P_{L}\right] D G^{+}+\text {h.c. }\right\} .(8
\end{aligned}
$$

Once again, we observe that the Higgs interactions are determined by basis-independent quantities (in this case, the quark masses, $\rho^{U}, \rho^{D}$ and $\beta-\alpha$ ). Thus, eq. (87) exhibits both flavor-changing Higgs-mediated neutral currents and CP-violating Higgs-fermion couplings (even though we assumed a CP-conserving Higgs potential, or equivalently the absence of tree-level mixing between the CP-odd $A$ and the CP-even $h$ and $H){ }^{18}$

For simplicity, we now focus on the case of one quark/lepton generation. Then, the Higgs-quark interaction produces the following Feynman rules of the form $-i g_{\phi f_{1} f_{2}}$ :

$$
\begin{gathered}
g_{h q \bar{q}}=\frac{m_{q}}{v} s_{\beta-\alpha}+\frac{1}{\sqrt{2}}\left(S_{q}+i \gamma_{5} P_{q}\right) c_{\beta-\alpha}, \\
g_{H q \bar{q}}=\frac{m_{q}}{v} c_{\beta-\alpha}-\frac{1}{\sqrt{2}}\left(S_{q}+i \gamma_{5} P_{q}\right) s_{\beta-\alpha},
\end{gathered}
$$

${ }^{18}$ In principle, this requires a fine-tuning of tree-level parameters. If $\operatorname{Im} \rho^{Q} \neq 0$, then one-loop effects will generate infinite corrections to CP-violating parameters of the Higgs potential (which are eliminated by renormalization of the Higgs potential parameters). The more general treatment in the case of a tree-level CP-violating Higgs potential will be addressed in a separate publication. 


$$
\begin{aligned}
g_{A u \bar{u}} & =-\frac{1}{\sqrt{2}}\left(i S_{u} \gamma_{5}-P_{u}\right), \\
g_{A d \bar{d}} & =\frac{1}{\sqrt{2}}\left(i S_{d} \gamma_{5}-P_{d}\right), \\
g_{H^{+} d \bar{u}} & =\frac{1}{2}\left[\rho^{D}\left(1+\gamma_{5}\right)-\rho^{U *}\left(1-\gamma_{5}\right)\right],
\end{aligned}
$$

where

$$
S_{q} \equiv \operatorname{Re} \rho^{Q}, \quad P_{q} \equiv \operatorname{Im} \rho^{Q}
$$

and $-i g_{H^{+} d \bar{u}}$ corresponds to the rule in which $d, \bar{u}$ and $H^{+}$are pointed into the vertex. As noted above, if $\operatorname{Im} \rho^{Q} \neq 0$, then the Higgs-fermion couplings are CP-violating.

The results of eqs. (87) and (88) apply to the most general type-III 2HDM. To study the Higgs-fermion interactions in type-I and type-II models, it is useful to have a basisindependent characterization of these two special patterns of Higgs couplings., This is formulated as follows: ${ }^{19}$

$$
\begin{aligned}
\left|\eta^{U}\right|^{2}\left|\eta^{D}\right|^{2}-\left|\eta^{U} \cdot \eta^{D}\right|^{2} & =0, & & \text { type-I } \\
\eta^{U} \cdot \eta^{D} & =0, & & \text { type-II } .
\end{aligned}
$$

To verify eqs. (94) and (95), simply note that these equations corresponds to the definitions of the type-I and type-II Higgs-fermion couplings in the special basis (i.e., for the particular value of $\beta$ ) in which two of the four Higgs-quark couplings of eq. (76) vanish. Since eqs. (94) and (95) are basis-independent conditions, they must be true if they are satisfied in one particular basis. Moreover, in both type-I and type-II models, the $\rho^{Q}$ are real. This is most easily proven in the special basis by using eq. (86) to show that the $\eta^{Q}$ must be real.

The type-I and type-II model conditions can be enforced by applying an appropriate discrete symmetry that distinguishes between $\Phi_{1}$ and $\Phi_{2}[10,11,12]$. Consequently, $\tan \beta$ is promoted to a physical parameter, and thus can be expressed in terms of invariant quantities. For example, in a type-II model, $\beta$ corresponds to the basis in which $\eta_{1}^{U}=\eta_{2}^{D}=0$. Using eq. (84), one obtains two different equations for $\tan \beta$ :

$$
\tan \beta=\frac{-\rho^{D}}{\kappa^{D}}=\frac{\kappa^{U}}{\rho^{U}} .
$$

\footnotetext{
${ }^{19}$ Note that the type-I condition can be written as $\left|\eta^{U} \times \eta^{D}\right|^{2}=0$, where $\times$ corresponds to the crossproduct. Since the Higgs flavor space is only two-dimensional, only one component of the cross-product exists. Nevertheless, it is tempting to write the type-I condition as $\eta^{U} \times \eta^{D}=0$.
} 
For these two definitions to be consistent, the following equation must be satisfied:

$$
\kappa^{U} \kappa^{D}+\rho^{U} \rho^{D}=0 .
$$

But, eq. (97) is equivalent to the type-II condition, $\eta^{U} \cdot \eta^{D}=0$, noted above. Moreover, using eqs. (85) and (196), it follows that:

$$
\rho^{D}=-\frac{\sqrt{2} m_{d}}{v} \tan \beta, \quad \rho^{U}=\frac{\sqrt{2} m_{u}}{v} \cot \beta .
$$

Inserting this result into eq. (87) [or eq. (88)] yields the well-known Feynman rules for the type-II Higgs-quark interactions. In deriving these results, the following trigonometric identities are particularly useful:

$$
\begin{aligned}
-\frac{\sin \alpha}{\cos \beta} & =s_{\beta-\alpha}-\tan \beta c_{\beta-\alpha}, & & \frac{\cos \alpha}{\sin \beta}=s_{\beta-\alpha}+\cot \beta c_{\beta-\alpha}, \\
\frac{\cos \alpha}{\cos \beta} & =c_{\beta-\alpha}+\tan \beta s_{\beta-\alpha}, & \frac{\sin \alpha}{\sin \beta} & =c_{\beta-\alpha}-\cot \beta s_{\beta-\alpha} .
\end{aligned}
$$

A similar analysis can be given for models of type-I.

The analysis above makes clear that $\tan \beta$ is a physical parameter in models of type-I and type-II, but is not meaningful in models of type-III. Nevertheless, it does suggest a strategy in the type-III CP-conserving case. ${ }^{20}$ Namely, one can introduce three $\tan \beta$-like parameters:

$$
\tan \beta_{d} \equiv \frac{-\rho^{D}}{\kappa^{D}}, \quad \tan \beta_{u} \equiv \frac{\kappa^{U}}{\rho^{U}}
$$

and a third parameter $\tan \beta_{e} \equiv-\rho^{E} / \kappa^{E}$ corresponding to the Higgs-lepton interaction. The meaning of these $\tan \beta$-like parameters is clear. For example, consider the case of up-type quark couplings to Higgs bosons of a type-III model. In the Higgs basis, both $\bar{Q}_{L}^{0} \widetilde{H}_{1} \kappa^{U, 0} U_{R}^{0}$ and $\bar{Q}_{L}^{0} \widetilde{H}_{2} \rho^{U, 0} U_{R}^{0}$ interaction terms are allowed. But, clearly there exists some basis (i.e., some rotation by angle $\beta_{u}$ from the Higgs basis) for which only one of the two up-type quark Yukawa couplings is non-vanishing. This defines the physical angle $\beta_{u}$, which is given in eq. (101). ${ }^{21}$ The angles $\beta_{u}, \beta_{d}$ and $\beta_{e}$ are physical parameters of the model, and in the

$\overline{20 \text { The } \rho^{Q}}$ are complex if CP-violating Higgs-fermion couplings are present, in which case the tan $\beta$-like parameters of eq. (101) would be complex. We shall treat the more general CP-violating case elsewhere. 21 Actually, there is a two-fold ambiguity in the definition of $\tan \beta$ corresponding to whether one identifies the Higgs boson that does not couple to the up-type quark as $\Phi_{1}$ or $\Phi_{2}$. The definition given in eq. (101) corresponds to the former case. In the latter case, one would define $\tan \beta_{u} \equiv-\rho^{U} / \kappa^{U}$. Said another way, suppose there exists some basis, corresponding to a rotation by an angle $\beta_{u}$ from the Higgs basis, for which one of the two up-type quark Yukawa couplings vanishes. Then a rotation from the Higgs basis by $\beta_{u}-\pi / 2$ will likewise yield one vanishing up-type quark Yukawa coupling. 
general type-III model there would be no reason for these parameters to be equal in value. However, in a type-II model, one would indeed find that $\tan \beta_{u}=\tan \beta_{d}=\tan \beta_{e}=\tan \beta$. In some cases, the Higgs sector is close to type-II. For example, the MSSM Higgs sector exhibits type-II couplings at tree-level, but all possible Higgs-fermion couplings appear at one-loop due to supersymmetry-breaking effects. In this case, the Higgs-fermion coupling is close to type-II, with differences among the three $\tan \beta$-like parameters introduced above generated by supersymmetry-breaking effects in loops corrections.

We can illustrate the last point in a very simple model approximation. In the MSSM at large $\tan \beta$ (and supersymmetric masses significantly larger than $m_{Z}$ ), the effective Lagrangian that describes the coupling of the Higgs bosons to the third generation quarks is given by:

$$
-\mathcal{L}_{\text {eff }}=h_{b}\left(\bar{q}_{L} \Phi_{1}\right) b_{R}+h_{t}\left(\bar{q}_{L} \widetilde{\Phi}_{2}\right) t_{R}+\Delta h_{b}\left(\bar{q}_{L} \Phi_{2}\right) b_{R}+\text { h.c. }
$$

where $\bar{q}_{L} \equiv\left(\bar{u}_{L}, \bar{d}_{L}\right)$. The term proportional to $\Delta h_{b}$ is generated at one-loop due to supersymmetry-breaking effects. ${ }^{22}$ The tree-level relation between $m_{b}$ and $h_{b}$ is modified [27]:

$$
m_{b}=\frac{h_{b} v}{\sqrt{2}} \cos \beta\left(1+\Delta_{b}\right)
$$

where $\Delta_{b} \equiv\left(\Delta h_{b} / h_{b}\right) \tan \beta$. That is, $\Delta_{b}$ is $\tan \beta$-enhanced, and governs the leading one-loop correction to the physical Higgs couplings to third generation quarks. In typical models at large $\tan \beta, \Delta_{b}$ can be of order 0.1 or larger and of either sign. ${ }^{23}$

In the approximation scheme above, eq. (84) yields $\kappa^{U} \simeq h_{t} \sin \beta, \rho^{U} \simeq h_{t} \cos \beta$, and

$$
\begin{aligned}
& \kappa^{D} \simeq h_{b} \cos \beta\left(1+\Delta_{b}\right) \\
& \rho^{D} \simeq-h_{b} \sin \beta\left(1-\frac{\Delta_{b}}{\tan ^{2} \beta}\right) \simeq-h_{b} \sin \beta .
\end{aligned}
$$

It follows that:

$$
\tan \beta_{d} \simeq \frac{\tan \beta}{1+\Delta_{b}}, \quad \tan \beta_{u} \simeq \tan \beta
$$

Thus, supersymmetry-breaking loop-effects can yield observable differences between $\tan \beta$ like parameters that are defined in terms of basis-independent quantities.

22 One-loop corrections to $h_{b}$ and $h_{t}$ and an effective (supersymmetry-breaking) operator $\Delta h_{t} q_{L} \widetilde{\Phi}_{1} t_{R}$ yield subdominant effects at large $\tan \beta$ and will therefore be neglected here.

${ }^{23}$ Explicit expressions for $\Delta_{b}$ in terms of supersymmetric masses and parameters, and references to the original literature can be found in ref. [5]. 
Finally, we briefly consider the multi-generation model. The type-III Higgs-quark interactions have already been given in eq. (87). Consider a type-II Higgs-quark interaction, defined by the matrix equations $\eta_{1}^{U}=\eta_{2}^{D}=0$. From eq. (84), it follows that the following two matrix equations must be satisfied:

$$
\boldsymbol{I} \tan \beta=-\rho^{D}\left(\kappa^{D}\right)^{-1}=\kappa^{U}\left(\rho^{U}\right)^{-1},
$$

where $\boldsymbol{I}$ is the identity matrix in quark flavor space. Again, we note that these equations are consistent because $\eta^{U} \cdot \eta^{D}=0$. Thus, using eq. (85),

$$
\rho^{D}=\frac{-\sqrt{2}}{v} M_{D} \tan \beta, \quad \rho^{U}=\frac{\sqrt{2}}{v} M_{U} \tan \beta,
$$

where $M_{D}$ and $M_{U}$ are the diagonal quark mass matrices (implying that $\rho^{D}$ and $\rho^{U}$ are also diagonal real matrices). We again conclude that $\tan \beta$ is a meaningful parameter. The analysis for type-I models proceeds in a similar manner. However, the construction of $\tan \beta$-like parameters in the general type-III model is far more complicated. In particular, although the $\kappa^{Q}$ are diagonal, the $\rho^{Q}$ are complex non-diagonal matrices in the general case. Thus, it does not seem very useful to define $\tan \beta$-like parameters by considering the non-diagonal matrices $\kappa^{U}\left(\rho^{U}\right)^{-1}$ and $-\rho^{D}\left(\kappa^{D}\right)^{-1}$. Fortunately, in most models, the thirdgeneration Yukawa couplings dominate, and one may define $\tan \beta$-like parameters based solely on the consideration of third-generation fermion couplings.

\section{SUMMARY}

The scalar Lagrangian of the two-Higgs-doublet model (2HDM) retains the same functional form under $2 \times 2$ unitary transformations among the two Higgs doublets. It is useful to understand the effect of these (unphysical) transformations on the Higgs potential parameters that govern the theory. These $\mathrm{U}(2)$ transformations can be thought of as generalized rotations among different bases of Higgs fields. The choice of basis is of course arbitrary.

In practice, one often chooses a basis in terms of vectors that arise naturally in the theory. For example, in the Higgs basis, the vacuum expectation value $v_{a}$ of the neutral Higgs fields points along the direction (in Higgs flavor space) of one of the two Higgs doublets. If one

defines $V_{a \bar{b}} \equiv v_{a} v_{b}^{*}$, then the Higgs basis corresponds to the orthonormal eigenvalues of $V_{a \bar{b}}$. In a CP-conserving theory, the CP-even Higgs squared-mass matrix is a $2 \times 2$ matrix 
whose orthonormal eigenvalues can be used to define the physical mass basis. The matrix of squared-masses, $Y_{a \bar{b}}$, which appears in the Higgs Lagrangian, can also be used to define a basis in which the off-diagonal term $Y_{12}=0$. When fermions are coupled to the Higgs sector, discrete symmetries are often imposed in order to guarantee the absence of flavor-changing neutral currents. These discrete symmetries are defined in a particular basis, which can be related to the structure of the Higgs-fermion interactions.

Although certain choices of basis may be physically motivated, any underlying assumption (e.g., the existence of a discrete symmetry, CP-invariance, etc.) must be experimentally tested. Thus, it is especially useful to analyze Higgs physics of the 2HDM independently of the choice of basis. Starting from an arbitrary (generic) basis, one can define invariant quantities - combinations of the Higgs potential parameters that are scalars with respect to $\mathrm{U}(2)$ transformations in the flavor space of the two Higgs doublets. Invariant descriptions of discrete symmetries and CP-symmetry can be used in principle to test for these symmetries. In addition, any physical Higgs sector observable (masses and couplings) can be expressed in terms of the $\mathrm{U}(2)$-invariants. In this paper, the relation of these observables to invariants has been obtained under the assumption that the Higgs scalar potential is CP-invariant. In this latter case, it is sufficient to consider restricted $\mathrm{O}(2)$-invariant quantities. In a subsequent publication, we will generalize the analysis given in sections [V] and $\nabla$ in order to treat the Higgs couplings in a model with a CP-violating 2HDM scalar potential.

In the most general $2 \mathrm{HDM}$, the ratio of the two vacuum expectation values, $\tan \beta$, is an unphysical basis-dependent quantity. Typically, $\tan \beta$ acquires meaning when the Higgsfermion interaction is formulated, since discrete symmetries or supersymmetry must be imposed in order to avoid flavor changing neutral currents in conflict with experiment. Nevertheless, these symmetries are typically broken symmetries, so within the effective theory of Higgs interactions, $\tan \beta$ again loses its meaning. In this paper, we have advocated replacing $\tan \beta$ with parameters that are more directly physical-invariant combinations of

Higgs-fermion Yukawa couplings. Once again, by focusing on U(2)-invariant quantities, one is led to a powerful and flexible formalism that is ideally suited for general phenomenological and theoretical studies of the two-Higgs-doublet model.

\section{Note Added}

After this work was completed, a paper of G.C. Branco, M.N. Rebelo and J.I. Silva- 
Marcos 28] appeared that emphasizes the techniques of invariants and addresses some of the issues considered in this paper.

\section{Acknowledgments}

This work was supported in part by the U.S. Department of Energy and the U.K. Particle Physics and Astronomy Research Council. We are grateful for many illuminating discussions with Jack Gunion. We have also benefited from conversations with Maria Krawczyk and Ilya Ginzburg. H.E.H. would like to express his gratitude to W.J. Stirling and the particle theory group in Durham for their warm hospitality during a three month sabbatical in January-March 2003 where this project was conceived.

\section{APPENDIX A: BASIS CHOICES FOR THE TWO-HIGGS DOUBLET MODEL}

In this appendix, we review the most general two-Higgs-doublet extension of the Standard Model [4, 14, 24]. Let $\Phi_{1}$ and $\Phi_{2}$ denote two complex $Y=1, \operatorname{SU}(2)_{L}$ doublet scalar fields. The most general gauge invariant scalar potential is given by

$$
\begin{aligned}
\mathcal{V}=m_{11}^{2} \Phi_{1}^{\dagger} \Phi_{1}+m_{22}^{2} \Phi_{2}^{\dagger} \Phi_{2}-\left[m_{12}^{2} \Phi_{1}^{\dagger} \Phi_{2}+\text { h.c. }\right] \\
+\frac{1}{2} \lambda_{1}\left(\Phi_{1}^{\dagger} \Phi_{1}\right)^{2}+\frac{1}{2} \lambda_{2}\left(\Phi_{2}^{\dagger} \Phi_{2}\right)^{2}+\lambda_{3}\left(\Phi_{1}^{\dagger} \Phi_{1}\right)\left(\Phi_{2}^{\dagger} \Phi_{2}\right)+\lambda_{4}\left(\Phi_{1}^{\dagger} \Phi_{2}\right)\left(\Phi_{2}^{\dagger} \Phi_{1}\right) \\
\quad+\left\{\frac{1}{2} \lambda_{5}\left(\Phi_{1}^{\dagger} \Phi_{2}\right)^{2}+\left[\lambda_{6}\left(\Phi_{1}^{\dagger} \Phi_{1}\right)+\lambda_{7}\left(\Phi_{2}^{\dagger} \Phi_{2}\right)\right] \Phi_{1}^{\dagger} \Phi_{2}+\text { h.c. }\right\}
\end{aligned}
$$

where $m_{11}^{2}, m_{22}^{2}$, and $\lambda_{1}, \cdots, \lambda_{4}$ are real parameters. In general, $m_{12}^{2}, \lambda_{5}, \lambda_{6}$ and $\lambda_{7}$ are complex. The scalar fields will develop non-zero vacuum expectation values if the mass matrix $m_{i j}^{2}$ has at least one negative eigenvalue. We assume that the parameters of the scalar potential are chosen such that the minimum of the scalar potential respects the $\mathrm{U}(1)_{\mathrm{EM}}$ gauge symmetry[29]. Then, the scalar field vacuum expectations values are of the form $^{24}$

$$
\left\langle\Phi_{1}\right\rangle=\frac{1}{\sqrt{2}}\left(\begin{array}{c}
0 \\
v_{1}
\end{array}\right), \quad\left\langle\Phi_{2}\right\rangle=\frac{1}{\sqrt{2}}\left(\begin{array}{c}
0 \\
v_{2} e^{i \xi}
\end{array}\right)
$$

\footnotetext{
24 In writing eq. (A2), we have used a global $\mathrm{SU}(2)_{W}$ rotation to put the non-zero vacuum expectation values in the lower component of the doublet, and a global hypercharge $\mathrm{U}(1)$ rotation to eliminate the phase of $v_{1}$.
} 
where $v_{1}$ and $v_{2}$ are real and non-negative, $0 \leq \xi<2 \pi$ and

$$
v^{2} \equiv v_{1}^{2}+v_{2}^{2}=\frac{4 m_{W}^{2}}{g^{2}}=(246 \mathrm{GeV})^{2}
$$

The corresponding potential minimum conditions are:

$$
\begin{gathered}
m_{11}^{2}=m_{12}^{2} e^{i \xi} t_{\beta}-\frac{1}{2} v^{2}\left[\lambda_{1} c_{\beta}^{2}+\left(\lambda_{3}+\lambda_{4}+\lambda_{5} e^{2 i \xi}\right) s_{\beta}^{2}+\left(2 \lambda_{6} e^{i \xi}+\lambda_{6}^{*} e^{-i \xi}\right) s_{\beta} c_{\beta}+\lambda_{7} s_{\beta}^{2} t_{\beta} e^{i \xi}\right](\mathrm{A} \\
m_{22}^{2}=\left(m_{12}^{2} e^{i \xi}\right)^{*} t_{\beta}^{-1}-\frac{1}{2} v^{2}\left[\lambda_{2} s_{\beta}^{2}+\left(\lambda_{3}+\lambda_{4}+\lambda_{5}^{*} e^{-2 i \xi}\right) c_{\beta}^{2}+\lambda_{6}^{*} c_{\beta}^{2} t_{\beta}^{-1} e^{-i \xi}\right. \\
\left.+\left(\lambda_{7} e^{i \xi}+2 \lambda_{7}^{*} e^{-i \xi}\right) s_{\beta} c_{\beta}\right]
\end{gathered}
$$

where

$$
s_{\beta} \equiv \sin \beta \equiv \frac{v_{2}}{v}, \quad c_{\beta} \equiv \cos \beta \equiv \frac{v_{1}}{v}, \quad \text { and } \quad t_{\beta} \equiv \tan \beta \equiv \frac{v_{2}}{v_{1}},
$$

and $0 \leq \beta \leq \pi / 2$ (since by assumption, $v_{1}, v_{2} \geq 0$ ). Since $m_{11}^{2}$ and $m_{22}^{2}$ are both real, the imaginary part of either eq. (A4) or eq. (A5) yields one independent equation:

$$
\operatorname{Im}\left(m_{12}^{2} e^{i \xi}\right)=\frac{1}{2} v^{2}\left[\operatorname{Im}\left(\lambda_{5} e^{2 i \xi}\right) s_{\beta} c_{\beta}+\operatorname{Im}\left(\lambda_{6} e^{i \xi}\right) c_{\beta}^{2}+\operatorname{Im}\left(\lambda_{7} e^{i \xi}\right) s_{\beta}^{2}\right]
$$

Eq. (A7) can be used to determine $\xi$.

Of the original eight scalar degrees of freedom, three Goldstone bosons $\left(G^{ \pm}\right.$and $\left.G^{0}\right)$ are absorbed ("eaten") by the $W^{ \pm}$and $Z$. These states are easily identified:

$$
\begin{aligned}
G^{ \pm} & =\cos \beta \Phi_{1}^{ \pm}+e^{-i \xi} \sin \beta \Phi_{2}^{ \pm} \\
G^{0} & =\sqrt{2}\left[\cos \beta \operatorname{Im} \Phi_{1}^{0}+\sin \beta \operatorname{Im}\left(e^{-i \xi} \Phi_{2}^{0}\right)\right] .
\end{aligned}
$$

In writing all the expressions above, we have implicitly chosen a basis in the space of $\Phi_{1}-\Phi_{2}$. We shall refer to this basis choice as the generic basis. One particular basis choice is especially useful. This is the so-called Higgs basis of ref. [15] (whose significance was also emphasized in refs. 99, 19, 22, 23]) in which only the neutral component of one of the two Higgs doublets $\left(e . g ., \Phi_{1}^{0}\right)$ possesses a vacuum expectation value. This requirement is not sufficient to uniquely define the Higgs basis. In particular, there is a set of $U(1)_{Y} \times U(1)$ transformations on the scalar field that preserves the conditions: $\left\langle\left\langle\Phi_{1}^{\prime 0}\right\rangle\right|=v / \sqrt{2}$ and $\left\langle\Phi_{2}^{\prime 0}\right\rangle=0$, where the $\Phi_{i}^{\prime}$ are the scalar fields in the basis where $\beta=0[\underline{6}] . \mathrm{U}(1)_{\mathrm{Y}}$ is the global hypercharge transformation, and is a symmetry of the Lagrangian, unlike the $\mathrm{SU}(2)$ transformations in Higgs flavor space which transform the parameters of the Higgs potential. The second U(1) is the diagonal subgroup of the $\mathrm{SU}(2)$ in Higgs flavor space, corresponding to $\Phi_{1}^{\prime} \rightarrow e^{i \chi} \Phi_{1}^{\prime}$ 
and $\Phi_{2}^{\prime} \rightarrow e^{-i \chi} \Phi_{1}^{\prime}$. Thus, there is a family of Higgs bases, parameterized by $\chi \cdot^{25}$ Let us denote the two Higgs doublets in the Higgs basis by $H_{1}$ and $H_{2}$. Then, the relation between these scalar doublet fields and the scalar fields in the original basis is given by:

$$
\begin{aligned}
e^{-i \chi} H_{1} & =\Phi_{1} \cos \beta+e^{-i \xi} \Phi_{2} \sin \beta \\
e^{i \chi} H_{2} & =-e^{i \xi} \Phi_{1} \sin \beta+\Phi_{2} \cos \beta .
\end{aligned}
$$

One then obtains

$$
e^{-i \chi} H_{1}=\left(\begin{array}{c}
G^{+} \\
\frac{1}{\sqrt{2}}\left(v+\varphi_{1}^{0}+i G^{0}\right)
\end{array}\right), \quad e^{i \chi} H_{2}=\left(\begin{array}{c}
H^{+} \\
\frac{1}{\sqrt{2}}\left(\varphi_{2}^{0}+i A\right)
\end{array}\right),
$$

where $\varphi_{1}^{0}, \varphi_{2}^{0}$ are CP-even neutral Higgs fields, $A$ is a CP-odd neutral Higgs field, and $H^{+}$ is the physical charged Higgs boson. If the Higgs sector is CP-violating, then $\varphi_{1}^{0}, \varphi_{2}^{0}$, and $A$ all mix to produce three physical neutral Higgs states of indefinite CP. If CP is conserved, then $A$ is the physical CP-odd Higgs scalar and $\varphi_{1}^{0}$ and $\varphi_{2}^{0}$ mix to produce two physical neutral CP-even Higgs states $h$ and $H$. We examine the CP-conserving case in more detail in section IV.

In the Higgs basis, the corresponding values of $\lambda_{1}, \cdots, \lambda_{7}$ can be easily computed by re-expressing $\Phi_{1}$ and $\Phi_{2}$ in terms of $H_{1}$ and $H_{2}$ [eq. (A10)] and inserting the result into eq. (A1). The end result is:

$$
\begin{aligned}
\mathcal{V}= & M_{11}^{2} H_{1}^{\dagger} H_{1}+M_{22}^{2} H_{2}^{\dagger} H_{2}-\left[M_{12}^{2} H_{1}^{\dagger} H_{2}+\text { h.c. }\right] \\
& +\frac{1}{2} \Lambda_{1}\left(H_{1}^{\dagger} H_{1}\right)^{2}+\frac{1}{2} \Lambda_{2}\left(H_{2}^{\dagger} H_{2}\right)^{2}+\Lambda_{3}\left(H_{1}^{\dagger} H_{1}\right)\left(H_{2}^{\dagger} H_{2}\right)+\Lambda_{4}\left(H_{1}^{\dagger} H_{2}\right)\left(H_{2}^{\dagger} H_{1}\right) \\
& +\left\{\frac{1}{2} \Lambda_{5}\left(H_{1}^{\dagger} H_{2}\right)^{2}+\left[\Lambda_{6}\left(H_{1}^{\dagger} H_{1}\right)+\Lambda_{7}\left(H_{2}^{\dagger} H_{2}\right)\right] H_{1}^{\dagger} H_{2}+\text { h.c. }\right\},
\end{aligned}
$$

where

$$
\begin{aligned}
M_{11}^{2} & =m_{11}^{2} c_{\beta}^{2}+m_{22}^{2} s_{\beta}^{2}-\operatorname{Re}\left(m_{12}^{2} e^{i \xi}\right) s_{2 \beta}, \\
M_{22}^{2} & =m_{11}^{2} s_{\beta}^{2}+m_{22}^{2} c_{\beta}^{2}+\operatorname{Re}\left(m_{12}^{2} e^{i \xi}\right) s_{2 \beta}, \\
M_{12}^{2} e^{i(\xi-2 \chi)} & =\frac{1}{2}\left(m_{11}^{2}-m_{22}^{2}\right) s_{2 \beta}+\operatorname{Re}\left(m_{12}^{2} e^{i \xi}\right) c_{2 \beta}+i \operatorname{Im}\left(m_{12}^{2} e^{i \xi}\right) .
\end{aligned}
$$

${ }^{25}$ The range of $\chi$ can be taken to be $0 \leq \chi \leq \pi$, since the transformation $\chi \rightarrow \chi+\pi$ can be compensated by a $\mathrm{U}(1)_{\mathrm{Y}}$ rotation. 
and

$$
\begin{aligned}
\Lambda_{1} & =\lambda_{1} c_{\beta}^{4}+\lambda_{2} s_{\beta}^{4}+\frac{1}{2} \lambda_{345} s_{2 \beta}^{2}+2 s_{2 \beta}\left[c_{\beta}^{2} \operatorname{Re}\left(\lambda_{6} e^{i \xi}\right)+s_{\beta}^{2} \operatorname{Re}\left(\lambda_{7} e^{i \xi}\right)\right] \\
\Lambda_{2}= & \lambda_{1} s_{\beta}^{4}+\lambda_{2} c_{\beta}^{4}+\frac{1}{2} \lambda_{345} s_{2 \beta}^{2}-2 s_{2 \beta}\left[s_{\beta}^{2} \operatorname{Re}\left(\lambda_{6} e^{i \xi}\right)+c_{\beta}^{2} \operatorname{Re}\left(\lambda_{7} e^{i \xi}\right)\right] \\
\Lambda_{3}= & \frac{1}{4} s_{2 \beta}^{2}\left[\lambda_{1}+\lambda_{2}-2 \lambda_{345}\right]+\lambda_{3}-s_{2 \beta} c_{2 \beta} \operatorname{Re}\left[\left(\lambda_{6}-\lambda_{7}\right) e^{i \xi}\right] \\
\Lambda_{4}= & \frac{1}{4} s_{2 \beta}^{2}\left[\lambda_{1}+\lambda_{2}-2 \lambda_{345}\right]+\lambda_{4}-s_{2 \beta} c_{2 \beta} \operatorname{Re}\left[\left(\lambda_{6}-\lambda_{7}\right) e^{i \xi}\right] \\
\Lambda_{5} e^{2 i(\xi-2 \chi)}= & \frac{1}{4} s_{2 \beta}^{2}\left[\lambda_{1}+\lambda_{2}-2 \lambda_{345}\right]+\operatorname{Re}\left(\lambda_{5} e^{2 i \xi}\right)+i c_{2 \beta} \operatorname{Im}\left(\lambda_{5} e^{2 i \xi}\right) \\
\Lambda_{6} e^{i(\xi-2 \chi)}= & -s_{2 \beta} c_{2 \beta} \operatorname{Re}\left[\left(\lambda_{6}-\lambda_{7}\right) e^{i \xi}\right]-i s_{2 \beta} \operatorname{Im}\left[\left(\lambda_{6}-\lambda_{7} c_{\beta}^{2}-\lambda_{2} s_{\beta}^{2}-\lambda_{345} c_{2 \beta}-i \operatorname{Im}\left(\lambda_{5} e^{2 i \xi}\right)\right]\right. \\
\Lambda_{7} e^{i(\xi-2 \chi)}= & -\frac{1}{2} s_{2 \beta}\left[\lambda_{1} s_{\beta}^{2}-\lambda_{2} c_{\beta}^{2}+\lambda_{345} c_{2 \beta}+i \operatorname{Im}\left(\lambda_{5} e^{2 i \xi}\right)\right] \\
& \quad+s_{\beta} s_{3 \beta} \operatorname{Re}\left(\lambda_{6} e^{i \xi}\right)+c_{\beta} c_{3 \beta} \operatorname{Re}\left(\lambda_{7} e^{i \xi}\right)+i s_{\beta}^{2} \operatorname{Im}\left(\lambda_{6} e^{i \xi}\right)+i c_{\beta}^{2} \operatorname{Im}\left(\lambda_{7} e^{i \xi}\right),
\end{aligned}
$$

where

$$
\lambda_{345} \equiv \lambda_{3}+\lambda_{4}+\operatorname{Re}\left(\lambda_{5} e^{2 i \xi}\right) .
$$

The inversion of eqs. (A13)-(A15) and eqs. (A16)-(A22) is simply obtained by making the replacements $M_{i j}^{2} \leftrightarrow m_{i j}^{2}, \Lambda_{i} \leftrightarrow \lambda_{i}, \beta \leftrightarrow-\beta$ and $\xi \leftrightarrow \xi-2 \chi$. Note that the Higgs basis corresponds to the choice $\beta=0$, independently of the value of $\xi$. The effect of non-uniqueness of the Higgs basis, which is parameterized by $\chi$, is also easily discerned. Starting from the Higgs basis with $\chi=0$, one can transform to the Higgs basis with arbitrary $\chi$ by the phase redefinitions

$$
H_{1} \rightarrow e^{i \chi} H_{1} \quad \text { and } \quad H_{2} \rightarrow e^{-i \chi} H_{2} .
$$

As a result of this transformation, the complex parameters of the scalar potential in the Higgs basis are transformed by a phase rotation: $M_{12}^{2}, \Lambda_{6}, \Lambda_{7} \rightarrow e^{2 i \chi}\left[M_{12}^{2}, \Lambda_{6}, \Lambda_{7}\right]$, and $\Lambda_{5} \rightarrow e^{4 i \chi} \Lambda_{5}$.

Finally, the scalar potential minimum conditions in Higgs basis are independent of $\chi$ :

$$
M_{11}^{2}=-\frac{1}{2} \Lambda_{1} v^{2}, \quad M_{12}^{2}=\frac{1}{2} \Lambda_{6} v^{2} .
$$

Note that the equation for $M_{12}^{2}$ is a complex equation that holds both for the real and imaginary parts. The value of $M_{22}^{2}$ is not constrained by the scalar potential minimum conditions. One can show that $M_{22}^{2}$ is directly related to the physical charged Higgs boson squared-mass:

$$
m_{H^{ \pm}}^{2}=M_{22}^{2}+\frac{1}{2} v^{2} \Lambda_{3},
$$


where $H^{ \pm}=-e^{i \xi} \sin \beta \Phi_{1}^{ \pm}+\cos \beta \Phi_{2}^{ \pm}$is the state orthogonal to the charged Goldstone boson [eq. (A8)]. The requirement that $m_{H^{ \pm}}^{2} \geq 0$ places a constraint on $M_{22}^{2}$ and $\Lambda_{3}$ since we have assumed that electromagnetism is not spontaneously broken.

We can now count the number of independent parameters that govern the most general 2HDM. After imposing the scalar potential minimum conditions in the Higgs basis, there are twelve parameters: $M_{11}^{2}$ (which determines the value of $v=246 \mathrm{GeV}$ [see eq. (A25)]), $M_{22}^{2}$, four real couplings $\Lambda_{1}, \ldots, \Lambda_{4}$ and three complex couplings $\Lambda_{5}, \Lambda_{6}$ and $\Lambda_{7}$. However, the non-uniqueness of the Higgs basis implies that only the relative phases of the these complex parameters are physical. That is, of the twelve independent parameters that characterize the Higgs basis, one degree of freedom can be removed by the phase redefinitions given by eq. (A24), leaving only eleven physical degrees of freedom [23].

To see that this result is consistent with the parameter counting in the generic basis, we first note that without loss of generality, we can choose a basis in which $m_{12}^{2}=0$. In this case, $\cos ^{2} 2 \beta$ becomes a physical parameter as noted at the end of section II. Moreover, we can make an additional phase rotation $\Phi_{2} \rightarrow e^{i \xi} \Phi_{2}$ such that the two neutral scalar vacuum expectation values are real. The minimum conditions [eqs. (A4) and (A5)] fix the values of $m_{11}^{2}$ and $m_{22}^{2}$, leaving twelve parameters: $v_{1}, v_{2}$, the real couplings $\lambda_{1}, \ldots, \lambda_{4}$ and the complex couplings $\lambda_{5}, \lambda_{6}$ and $\lambda_{7}$. But, in the basis where $m_{12}^{2}=0$, the imaginary part of the minimum conditions [eq. (A7)] implies one relation among $\operatorname{Im} \lambda_{5}, \operatorname{Im} \lambda_{6}$ and $\operatorname{Im} \lambda_{7}$. Thus, we again end up with eleven independent parameters, as expected. We may also obtain the same result by employing an elegant technique advocated in refs. [30] and 31]. In the generic basis, the scalar Higgs potential consists of six real and four complex parameters. If the Higgs potential is set to zero, the scalar Lagrangian possesses a $\mathrm{U}(2)$ global symmetry corresponding to arbitrary U(2) transformations in the "flavor" space of Higgs doublets $\left\{\Phi_{1}, \Phi_{2}\right\}$. In particular, note that $\mathrm{U}(2) \cong \mathrm{SU}(2) \times \mathrm{U}(1)_{\mathrm{Y}}$, where the $\mathrm{SU}(2)$ is a global flavor symmetry not to be confused with the gauged SU(2) of the Standard Model. When the Higgs potential is restored, the scalar Lagrangian is no longer invariant under the $\mathrm{SU}(2)$ global flavor transformations. That is, the effect of the flavor SU(2) transformations is to transform the Higgs potential parameters. However, we are always free to redefine the scalar fields by the flavor SU(2) transformation. A general SU(2) transformation is determined by three parameters. Thus, three of the fourteen Higgs potential parameters can be transformed away (or set to zero) by an appropriate flavor SU(2) redefinition of the 
two scalar doublet fields. This leaves eleven physical degrees of freedom, again confirming our previous counting.

\section{APPENDIX B: INVARIANTS BY EXPLICIT CONSTRUCTION OF EIGEN- VECTORS}

In this Appendix, we explicitly construct eigenvectors of the hermitian matrix $V_{a \bar{b}} \equiv \widehat{v}_{a} \widehat{v}_{\bar{b}}^{*}$ [where $\widehat{v}$ is the unit vector of Higgs vacuum expectation values defined in eq. (17)], and contract them with the Higgs potential parameter tensors $(Y$ and $Z$ ) to obtain index-free "objects". By carefully considering the phase transformations on the eigenvectors, we will see that some of our objects are invariants with respect to $\mathrm{U}(2)$, and some pick up phases under $\mathrm{U}(2)$ transformations. The latter set can be combined into the invariants of section II.

Consider the two orthonormal eigenvectors of $V$ :

$$
\widehat{v}_{a} \equiv e^{i \eta}\left(\begin{array}{c}
c_{\beta} \\
s_{\beta} e^{i \xi}
\end{array}\right), \quad \widehat{w}_{a} \equiv-\epsilon_{a b} \widehat{v}_{\bar{b}}^{*}=e^{-i \eta}\left(\begin{array}{c}
-s_{\beta} e^{-i \xi} \\
c_{\beta}
\end{array}\right)
$$

with the inverse relation $\widehat{v}_{\bar{a}}^{*}=\epsilon_{\bar{a} \bar{b}} w_{b}$. In eqs. (7) and (A2), a global $\mathrm{U}(1)_{Y}$ rotation has been employed to eliminate the overall phase factor $e^{i \eta}$ in $\widehat{v}$. However, it will sometimes be convenient to use the more general forms given in eq. (B1).

Given a generic basis and the corresponding form for $\widehat{v}$, we can transform to another basis by redefining the scalar fields via $\Phi_{a}^{\prime}=U_{a \bar{b}} \Phi_{b}$. The most general $\mathrm{U}(2)$ transformation is given by

$$
U=e^{i \psi}\left(\begin{array}{cc}
e^{i \gamma} \cos \theta & e^{-i \zeta} \sin \theta \\
-e^{i \zeta} \sin \theta & e^{-i \gamma} \cos \theta
\end{array}\right)
$$

where $0 \leq \theta \leq \pi / 2$, and $0 \leq \gamma \leq \pi$ and $0 \leq \zeta, \psi<2 \pi{ }^{26}$ The subgroup of $\mathrm{U}(1)_{\mathrm{Y}}$ global hypercharge rotations corresponds to freezing the values of $\theta, \gamma$ and $\zeta$, and the subgroup of the flavor $\mathrm{SU}(2)$ transformations corresponds to freezing the value of $\psi$. Starting from the generic basis, we first make a $\mathrm{U}(1)_{\mathrm{Y}}$ transformation to set $\eta=0$ in eq. (B1). Then, we may employ the flavor $\mathrm{SU}(2)$ transformation given by $\psi=0, \gamma=\chi, \zeta=\xi-\chi$ and $\theta=\beta$

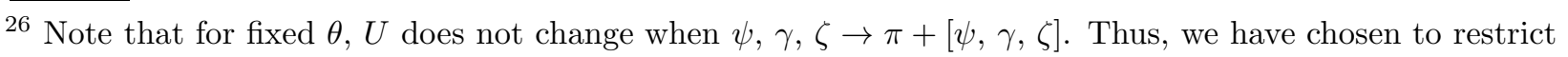
$\chi$ to lie between 0 and $\pi$ (other choices are possible). If $\psi=0$, then eq. (B2) provides a parameterization of $\mathrm{SU}(2) / \mathbf{Z}_{\mathbf{2}}$. In this case, the full $\mathrm{SU}(2)$ group manifold can be covered by extending the range of $\gamma$ to $0 \leq \gamma<2 \pi$. 
to rotate from the generic basis to the Higgs basis as specified by eq. (A10). Applying this form of $U$ to $\widehat{v}$ and $\widehat{w}$ [eq. (B1) with $\eta=0$ ] yields the corresponding results in the Higgs basis: $\widehat{v}=\left(e^{i \chi}, 0\right)$ and $\widehat{w}=\left(0, e^{-i \chi}\right)$. The parameter $\chi$ reflects the non-uniqueness in the definition of the Higgs basis, as discussed in Appendix A

We now turn to the task of constructing U(2)-invariant (scalar) combinations of Higgs potential parameters. We shall also construct objects that are invariant with respect to the flavor $\mathrm{SU}(2)$ transformations, but not necessarily invariant with respect to the full $\mathrm{U}(2)$ group. These objects are useful, but they do not correspond directly to physical quantities.

We may combine the tensors $Y_{a \bar{b}}, Z_{a \bar{b}} c \bar{d}, \widehat{v}_{a}, \widehat{v}_{\bar{a}}^{*}, \widehat{w}_{a}$ and $\widehat{w}_{\bar{a}}^{*}$ to create scalar quantities by summing over pairs of indices (following the rule that the summed index pairs must contain one unbarred and one barred index). A U(2) transformation can be written as $U \equiv e^{i \psi} \widehat{U}$ with det $\widehat{U}=1$. With respect to the flavor-SU(2), both $\widehat{v}$ and $\widehat{w}$ transform covariantly: ${ }^{27}$ $\widehat{v}_{a} \rightarrow \widehat{U}_{a \bar{b}} \widehat{v}_{b}$ then $\widehat{w}_{a} \rightarrow \widehat{U}_{a \bar{b}} \widehat{w}_{b}$. With respect to $\mathrm{U}(1)_{\mathrm{Y}}, \widehat{v}$ inherits the transformation law of $\Phi_{a}$. Hence with respect to $\mathrm{U}(2)$ transformations, $\widehat{v}_{a} \rightarrow U_{a \bar{b}} \widehat{v}_{b}$. In contrast,

$$
\widehat{w}_{a} \rightarrow(\operatorname{det} U)^{-1} U_{a \bar{b}} \widehat{w}_{b}
$$

That is, $\widehat{v}$ and $\widehat{w}$ transform oppositely with respect to $\mathrm{U}(1)_{\mathrm{Y}}$ as expected.

It is now straightforward to construct index-free objects that are linear in the Higgs potential parameters. We obtain three squared-mass invariants:

$$
Y_{1} \equiv Y_{a \bar{b}} \widehat{v}_{\bar{a}}^{*} \widehat{v}_{b}, \quad Y_{2} \equiv Y_{a \bar{b}} \widehat{w}_{\bar{a}}^{*} \widehat{w}_{b}, \quad Y_{3} \equiv Y_{a \bar{b}} \widehat{v}_{\bar{a}}^{*} \widehat{w}_{b}
$$

and seven coupling invariants:

$$
\begin{aligned}
& Z_{1} \equiv Z_{a \bar{b} c \bar{d}} \widehat{v}_{\bar{a}}^{*} \widehat{v}_{b} \widehat{v}_{\bar{c}}^{*} \widehat{v}_{d}, \quad Z_{2} \equiv Z_{a \bar{b} c \bar{d}} \widehat{w}_{\bar{a}}^{*} \widehat{w}_{b} \widehat{w}_{\bar{c}}^{*} \widehat{w}_{d}, \\
& Z_{3} \equiv Z_{a \bar{b} c \bar{d}} \widehat{v}_{\bar{a}}^{*} \widehat{v}_{b} \widehat{w}_{\bar{c}}^{*} \widehat{w}_{d}, \quad Z_{4} \equiv Z_{a \bar{b} c \bar{d}} \widehat{w}_{\bar{a}}^{*} \widehat{v}_{b} \widehat{v}_{\bar{c}}^{*} \widehat{w}_{d}, \\
& Z_{5} \equiv Z_{a \bar{b} c \bar{d}} \widehat{v}_{\bar{a}}^{*} \widehat{w}_{b} \widehat{v}_{\bar{c}}^{*} \widehat{w}_{d}, \\
& Z_{6} \equiv Z_{a \bar{b} c \bar{d}} \widehat{v}_{\bar{a}}^{*} \widehat{v}_{b} \widehat{v}_{\bar{c}}^{*} \widehat{w}_{d}, \\
& Z_{7} \equiv Z_{a \bar{b} c \bar{d}} \widehat{v}_{\bar{a}}^{*} \widehat{w}_{b} \widehat{w}_{\bar{c}}^{*} \widehat{w}_{d} \text {. }
\end{aligned}
$$

27 The fact that $\widehat{w}$ transforms covariantly under $\mathrm{SU}(2)$ transformations, $\widehat{U}$, is a consequence of the wellknown identity among Pauli matrices: $\sigma_{2} \overrightarrow{\boldsymbol{\sigma}} \sigma_{2}=\overrightarrow{\boldsymbol{\sigma}}^{*}$. This identity can be used to prove the relation $\epsilon_{a b} \widehat{U}_{c \bar{b}}^{\dagger}=\widehat{U}_{a \bar{b}} \epsilon_{b c}$. 
Noting the transformation law for $w$ [eq. (B33)], it then follows that the real quantities $Y_{1}, Y_{2}, Z_{1,2,3,4}$ are $\mathrm{U}(2)$-invariants, whereas the complex quantities $Y_{3}$ and $Z_{5,6,7}$ are only flavor-SU(2)-invariants. However, from the four complex flavor-SU(2)-invariants, we can construct seven real $\mathrm{U}(2)$-invariant quantities: the magnitudes $\left|Y_{3}\right|$ and $\left|Z_{5,6,7}\right|$, and three relative phases: $\arg \left(Y_{3}^{2} Z_{5}^{*}\right), \arg \left(Y_{3} Z_{6}^{*}\right)$ and $\arg \left(Y_{3} Z_{7}^{*}\right)$. Thus, we have recovered the thirteen $\mathrm{U}(2)$-invariant quantities given in eqs. (14)-(23). Imposing the scalar potential minimum conditions [eqs. (30) and (31)] reduces the number of physical degrees of freedom to eleven as expected.

Let us first evaluate eqs. (Bי $-(\underline{B} 9)$ in the Higgs basis where $\widehat{v}=\left(e^{i \chi}, 0\right)$ and $\widehat{w}=\left(0, e^{-i \chi}\right)$. By inspection, one obtains

$$
\begin{aligned}
& Y_{1}=M_{11}^{2}, \quad Y_{2}=M_{22}^{2}, \quad Y_{3}=-M_{12}^{2} e^{-2 i \chi}, \quad Z_{i}=\Lambda_{i} \quad(i=1, \ldots, 4), \\
& Z_{5}=\Lambda_{5} e^{-4 i \chi}, \quad Z_{6}=\Lambda_{6} e^{-2 i \chi}, \quad Z_{7}=\Lambda_{7} e^{-2 i \chi} .
\end{aligned}
$$

The appearance of the $\chi$-dependent phases in the expressions for the complex invariants in eq. (B10) is expected. As noted below eq. (A24), if one transforms from the Higgs basis with $\chi=0$ to a Higgs basis with arbitrary $\chi$, then $M_{12}^{2}, \Lambda_{6}, \Lambda_{7} \rightarrow e^{2 i \chi}\left[M_{12}^{2}, \Lambda_{6}, \Lambda_{7}\right]$, and $\Lambda_{5} \rightarrow e^{4 i \chi} \Lambda_{5}$. But, this is precisely what is needed to ensure that $Y_{3}$ and $Z_{5,6,7}$ are invariant with respect to the flavor-SU(2) transformation $U_{D} \equiv \operatorname{diag}\left(e^{i \chi}, e^{-i \chi}\right)$. One can also evaluate eqs. (B4) $-(\mathrm{B} 9)$ in the generic basis where $\widehat{v}$ and $\widehat{w}$ are given by eq. (B1) with $\eta=0$. The result of this computation simply reproduces the results of eqs. (A13)-(A22).

Using eqs. (21) and (41), one can show that eqs. (B4)-(B9) exhaust all possible independent invariants that are linear in the Higgs potential parameters. For example, by inserting $\widehat{w}_{a}=-\epsilon_{a b} \widehat{v}_{b}^{*}$ into eqs. (B-

$$
\begin{aligned}
Y_{1}+Y_{2} & =\operatorname{Tr} Y, \\
Z_{1}+Z_{3} & =Z_{a \bar{b}}^{(2)} v_{\bar{a}}^{*} v_{b}, \\
Z_{1}+Z_{4} & =Z_{a \bar{b}}^{(1)} v_{\bar{a}}^{*} v_{b}, \\
Z_{1}+Z_{2}+2 Z_{3} & =\operatorname{Tr} Z^{(2)}, \\
Z_{1}+Z_{2}+2 Z_{4} & =\operatorname{Tr} Z^{(1)},
\end{aligned}
$$

which demonstrates that $Z_{a \bar{b}}^{(k)} v_{\bar{a}}^{*} v_{b}$ and $\operatorname{Tr} Z^{(k)}(k=1,2)$ are not independent invariants. Note that the latter two invariant quantities are independent of the vacuum expectation 
values, and thus correspond to combinations of the Higgs self couplings that are invariant under an arbitrary change of basis. ${ }^{28}$ Furthermore, $\epsilon_{\bar{a} \bar{c}} \epsilon_{b d} Z_{a \bar{b} c \bar{d}}=\operatorname{Tr}\left[Z^{(2)}-Z^{(1)}\right]=\lambda_{3}-\lambda_{4}$ is not independent from the invariants of eqs. (B14) and (B15) ${ }^{29}$

If we restrict our considerations to a CP-conserving theory, where all scalar coupling parameters may be taken real and basis changes are restricted to those related by $\mathrm{O}(2)$ transformations, then the distinction between barred and unbarred indices becomes irrelevant. In this case, one more independent invariant arises that is linear in the couplings: $Z_{a b a b}=\lambda_{1}+\lambda_{2}+2 \lambda_{5}$, with respect to any real basis choice. ${ }^{30}$

Likewise, one can also construct additional invariants involving higher powers of the mass terms $Y$ and the couplings $Z$. For example, $2 \times 2$ matrices satisfy

$$
\operatorname{det} Y=\frac{1}{2} \epsilon_{b d} \epsilon_{\bar{a} \bar{c}} Y_{a \bar{b}} Y_{c \bar{d}}=\frac{1}{2}\left[(\operatorname{Tr} Y)^{2}-\operatorname{Tr} Y^{2}\right] .
$$

This yields the (invariant) determinant. One can also construct invariants that depend on inverse powers of $Y$ [since $Y^{-1}$ transforms the same way as $Y$ under $\mathrm{U}(2)$ ]. However, according to the Cayley-Hamilton theorem, all matrices satisfy their characteristic equations [32]. Consequently, $(\operatorname{det} Y)\left[Y^{-1}\right]_{a \bar{b}}=(\operatorname{Tr} Y) \delta_{a \bar{b}}-Y_{a \bar{b}}$. Thus, it is sufficient to consider invariants constructed out of positive powers of $Y$. One can define a number of different inverses for $Z$ depending on how one sums over the repeated indices. Nevertheless, a similar conclusion holds and one can restrict considerations to invariants involving positive powers of $Z$.

As a final application of these techniques, we briefly discuss the $\mathbf{Z}_{\mathbf{2}}$ discrete symmetry $\Phi_{1} \rightarrow+\Phi_{1}, \Phi_{2} \rightarrow-\Phi_{2}$, which implies that $m_{12}^{2}=\lambda_{6}=\lambda_{7}=0$ in some basis. In section we have exhibited $\mathrm{U}(2)$-invariant (basis-independent) conditions in terms of commutators of Higgs potential coupling second-rank tensors that imply the existence of a basis where the $\mathbf{Z}_{\mathbf{2}}$ discrete symmetry is manifest. Here, we provide another method for constructing $\mathrm{U}(2)$-invariant conditions. In this method, we introduce the two orthonormal eigenvectors of $Y_{a b}$, which we shall denote by $\widehat{y}$ and $\widehat{z}$. It is convenient to define $\widehat{z}_{a} \equiv-\epsilon_{a b} y_{\bar{b}}^{*}$, since $\widehat{z}_{a} \widehat{y}_{\bar{a}}^{*}=0$.

${ }^{28}$ That is, $\operatorname{Tr} Z^{(2)} \equiv Z_{a \bar{a} b \bar{b}}=\lambda_{1}+\lambda_{2}+2 \lambda_{3}$ and $\operatorname{Tr} Z^{(1)} \equiv Z_{a \bar{b} b \bar{a}}=\lambda_{1}+\lambda_{2}+2 \lambda_{4}$, with respect to any basis choice.

${ }^{29}$ Once can also define $X_{a b} \equiv \epsilon_{c d} Z_{a \bar{c} b \bar{d}}$. However, employing this tensor does not lead to any genuinely new invariants. One can easily verify that $X_{a b}=\left(\lambda_{3}-\lambda_{4}\right) \epsilon_{a b}$, which we recognize as an SU(2)-invariant tensor.

30 Note that eqs. A16) yield the relation $\Lambda_{1}+\Lambda_{2}+2 \operatorname{Re}\left(\Lambda_{5} e^{2 i(\xi-2 \chi)}\right)=\lambda_{1}+\lambda_{2}+2 \operatorname{Re}\left(\lambda_{5} e^{2 i \xi}\right)$. However, one cannot extract from this result a quantity that is invariant under the most general flavor $\mathrm{SU}(2)$ and $\mathrm{U}(2)$ transformations. 
It then follows that:

$$
Y_{a \bar{b}} \widehat{y}_{\bar{a}}^{*} \widehat{z}_{b}=0
$$

That is, $m_{12}^{2}=0$ in any basis where $\widehat{y}=\left(e^{i \chi_{y}}, 0\right)$. The freedom to vary $0 \leq \chi_{y} \leq \pi$ corresponds to the simple fact that if $m_{12}^{2}=0$ in some basis $\Phi_{a}$, then $m_{12}^{2}=0$ is also zero in the rotated basis $\left(U_{D}\right)_{a \bar{b}} \Phi_{b}$, where $U_{D}=\operatorname{diag}\left(e^{i \chi_{y}}, e^{-i \chi_{y}}\right)$. Eq. (B17) also implies that $\widehat{y}$ and $\widehat{z}$ transform covariantly with respect to the flavor-SU(2). To derive this result, we write $U=e^{i \psi} \widehat{U}$ as before, where $\operatorname{det} \widehat{U}=1$. Using the $\mathrm{U}(2)$ transformation law satisfied by $Y_{a \bar{b}}$, it follows that $\widehat{y}$ and $\widehat{z}$ transform as $\widehat{y} \rightarrow \widehat{U} \widehat{y}$ and $\widehat{z} \rightarrow \widehat{U} \widehat{z}$. The behavior of $\widehat{y}_{a}$ and $\widehat{z}_{a}$ under $\mathrm{U}(1)_{\mathrm{Y}}$ transformations is arbitrary. ${ }^{31}$ Independently of the behavior of $\widehat{y}$ and $\widehat{z}$ under $\mathrm{U}(1)_{\mathrm{Y}}$, eq. (B17) is a $\mathrm{U}(2)$-invariant equation since any overall phase of $\widehat{y}_{\bar{a}}^{*} \widehat{z}_{b}$ simply drops out of the equation.

Eq. (B17) can be used to derive the value of $\cos ^{2} 2 \beta$, where $\widehat{v}=\left(c_{\beta}, s_{\beta} e^{i \xi}\right)$ in the eigenbasis of $Y$ (i.e., the basis where $Y$ is diagonal). In this basis, we may take $\widehat{y}=(1,0)$ and $\widehat{z}=(0,1)$. Transforming to the Higgs basis [eq. (A10) $]$, one finds that $\widehat{y}=\left(e^{i \chi} c_{\beta},-e^{i(\xi-\chi)} s_{\beta}\right), \widehat{z}=$ $\left(e^{-i(\xi-\chi)} s_{\beta}, e^{-i \chi} c_{\beta}\right)$ and $\widehat{v}=\left(e^{i \chi}, 0\right)$. Thus, evaluating eq. (B17) in the Higgs basis, we obtain:

$$
\left(Y_{22}-Y_{11}\right) s_{\beta} c_{\beta}=Y_{12} e^{i(\xi-2 \chi)} c_{\beta}^{2}-Y_{12}^{*} e^{-i(\xi-2 \chi)} s_{\beta}^{2} .
$$

However, eq. A15 implies that $Y_{12} e^{i(\xi-2 \chi)}$ is real. Thus, we may write $Y_{12} e^{i(\xi-2 \chi)}= \pm\left|Y_{12}\right|$, where the choice of sign reflects two possible transformations from the eigenbasis of $Y$ to the Higgs basis (which differ by the interchange of $\Phi_{1}$ and $\Phi_{2}$ in the eigenbasis of $Y$ ). Consequently, eq. (B18) implies that

$$
\frac{c_{2 \beta}^{2}}{s_{2 \beta}^{2}}=\frac{\left(Y_{11}-Y_{22}\right)^{2}}{4\left|Y_{12}\right|^{2}},
$$

which is equivalent to the result previously given for $\cos ^{2} 2 \beta$ [see eq. (34)] evaluated in the Higgs basis.

The basis-independent conditions for the $\Phi_{1} \rightarrow+\Phi_{1}, \Phi_{2} \rightarrow-\Phi_{2}$ discrete symmetry are given by:

$$
\begin{aligned}
& Z_{a \bar{b} c \bar{d}} \widehat{y}_{\bar{a}}^{*} \widehat{y}_{b} \widehat{y}_{\bar{c}}^{*} \widehat{z}_{d}=0, \\
& Z_{a \bar{b} c \bar{d}} \widehat{z}_{\bar{a}}^{*} \widehat{z}_{b} \widehat{z}_{\bar{c}}^{*} \widehat{y}_{d}=0 .
\end{aligned}
$$

${ }^{31}$ Since $Y_{a \bar{b}}$ is invariant with respect to $\mathrm{U}(1)_{\mathrm{Y}}$, the eigenvalue equations that defines $\widehat{y}$ and $\widehat{z}$ are unchanged. The orthonormal eigenvectors are always defined only up to an arbitrary phase. 
As above, any overall phase redefinitions of $\widehat{y}$ and $\widehat{z}$ have no effect on eqs. (B20) and (B21), which ensures that these are $\mathrm{U}(2)$-invariant conditions.

One can use these results to obtain conditions that depend only on invariant combinations of Higgs potential parameters. As above, our strategy is to evaluate eqs. (B20) and (B21) in the Higgs basis. The outcome of this computation is two independent relations among invariant combinations of Higgs basis parameters. The details are not very illuminating, so we omit them here. One can check that evaluating the commutator conditions given in section $\amalg \mathrm{B}$ in the Higgs basis produces equivalent results.

Suppose that in the basis where the discrete symmetry $\Phi_{1} \rightarrow+\Phi_{1}, \Phi_{2} \rightarrow-\Phi_{2}$ is manifest, one also has $\lambda_{5}=0$. Then, the discrete symmetry is promoted to a global U(1) Peccei-Quinn symmetry corresponding to $\Phi_{1} \rightarrow e^{i \alpha} \Phi_{1}, \Phi_{2} \rightarrow e^{-i \alpha} \Phi_{2}$. This symmetry is spontaneously broken by the scalar field vacuum expectation values. Consequently, the (tree-level) mass of the CP-odd Higgs scalar (which is to be identified with the axion [33]) must vanish. The basis-independent conditions for the existence of the Peccei-Quinn symmetry are given by eqs. (B17) -(B21) supplemented by a fourth condition:

$$
Z_{a \bar{b} c \bar{d}} \widehat{y}_{\bar{a}}^{*} \widehat{z}_{b} \widehat{y}_{\bar{c}}^{*} \widehat{z}_{d}=0
$$

Again, we may evaluate eq. (B22) in the Higgs basis. Combining this result with the corresponding relations obtained from eqs. (B17)-(B21), it is possible to prove [using eqs. (A26) and (68)] that $m_{A}^{2}=0$, which identifies $A$ as the axion.

\section{APPENDIX C: DISCOVERING A DISCRETE SYMMETRY}

In ref. [26], a $2 \mathrm{HDM}$ is presented that possesses a permutation symmetry in which the scalar Lagrangian is invariant under the interchange of $\Phi_{1}$ and $\Phi_{2}$ in the generic basis. As a result of the permutation symmetry, it follows that ${ }^{32}$

$$
m_{11}^{2}=m_{22}^{2}, \lambda_{1}=\lambda_{2} \text {, and } \lambda_{7}=\lambda_{6}^{*} \text {, with } m_{12}^{2} \text { and } \lambda_{5} \text { real. }
$$

In this model, we shall verify that the commutator conditions [eqs. (39) and (40)] of section

32 The authors of ref. 26] assume in addition that $\lambda_{6}$ is real, although this is not required by the permutation symmetry. 
$\Phi_{1}^{\prime} \rightarrow \Phi_{1}^{\prime}$ and $\Phi_{2}^{\prime} \rightarrow-\Phi_{2}^{\prime}$ in some new basis $\Phi^{\prime}$. Inserting eq. (C1) into the definitions of $Y$, $Z^{(1)}$ and $Z^{(11)}$, one can check that the two diagonal elements of each of the three matrices are equal. Similarly, the two off-diagonal elements of each of the three matrices are equal. All $2 \times 2$ matrices with these properties commute. Thus, eqs. (391) and (40) are verified.

Although the invariant conditions provide a powerful method for analysis, this particular model is simple enough for pedestrian methods. In particular, starting from from the $\Phi$-basis that respects the $\Phi_{1} \leftrightarrow \Phi_{2}$ permutation symmetry, one may employ eq. (B2) to transform to a new $\Phi^{\prime}$-basis. Then, the new basis respects the $\Phi_{1}^{\prime} \rightarrow \Phi_{1}^{\prime}, \Phi_{2}^{\prime} \rightarrow-\Phi_{2}^{\prime}$ discrete symmetry if $\theta=\pi / 4$ and $\zeta+\gamma=0$. One can now check that for $\xi=0$, the $\Phi^{\prime}$-basis corresponds to the Higgs basis, while for $\xi \neq 0$ and $\lambda_{6}$ real, the $\Phi^{\prime}$-basis is related to the Higgs basis by a rotation of $\beta=\xi / 2$ or $\beta=(\pi-\xi) / 2$.

Having identified the $\mathbf{Z}_{\mathbf{2}}$ discrete symmetry, it follows that the model is CP-conserving. This conclusion is less transparent in the original basis [where eq. (C1) is satisfied]. Consider that one solution to the scalar potential minimum conditions [eqs. (A4) and (A5)] is $\beta=\pi / 4$. By subtracting the two minimum conditions, one finds that

$$
\left[m_{12}^{2}-\frac{1}{2} v^{2}\left(\lambda_{5} \cos \xi+\lambda_{6}\right)\right] \sin \xi=0
$$

Two cases can be examined. If $\operatorname{Im} \lambda_{6} \neq 0$, then the only solution to eq. (C2) is $\xi=0(\bmod \pi)$. If $\lambda_{6}$ is real, then a second solution for $\xi$ exists, $\cos \xi=\left(2 m_{12}^{2}-\lambda_{6} v^{2}\right) /\left(\lambda_{5} v^{2}\right)$, which implies that the vacuum expectation value has a non-trivial complex phase. In this case, one might naively assume that $\mathrm{CP}$ is spontaneously broken, but ref. [26] shows that this is a false conclusion.

It is noteworthy that in first case above, $\lambda_{6}$ was complex in a basis where all other scalar potential parameters and the vacuum expectation value are real, and in the second case above, the vacuum expectation values exhibited a relative phase in a basis where all Higgs potential parameters are real. Nevertheless, it is straightforward to check that in both cases, the three CP-odd invariants $I_{1}, I_{2}$ and $I_{3}$ [eq. (158)] are real. Indeed a 2HDM model that respects a permutation symmetry $\Phi_{1} \leftrightarrow \Phi_{2}$ is explicitly CP-conserving, and CP is not spontaneously broken by the vacuum.

[1] T.D. Lee, Phys. Rev. D8 1226 (1973); Phys. Rep. 9, 143 (1974). 
[2] R.D. Peccei and H.R. Quinn, Phys. Rev. Lett. 38, 1440 (1977).

[3] P. Fayet, Nucl. Phys. B78, 14 (1974); B90, 104 (1975); K. Inoue, A. Kakuto, H. Komatsu and S. Takeshita, Prog. Theor. Phys. 67, 1889 (1982); R.A. Flores and M. Sher, Annals Phys. (NY) 148, 95 (1983); J.F. Gunion and H.E. Haber, Nucl. Phys. B272, 1 (1986) [Erratum: B402, 567 (1993)].

[4] J.F. Gunion, H.E. Haber, G. Kane and S. Dawson, The Higgs Hunter's Guide (Perseus Publishing, Cambridge, MA, 1990).

[5] M. Carena and H.E. Haber, Prog. Part. Nucl. Phys. 50, 63 (2003) arXiv:hep-ph/0208209.

[6] G.C. Branco, L. Lavoura and J.P. Silva, CP Violation (Oxford University Press, Oxford, England, 1999), chapters 22 and 23.

[7] P. Fayet and S. Ferrara, Phys. Rept. 32, 249 (1977); H.P. Nilles, Phys. Rep. 110, 1 (1984); H.E. Haber and G. L. Kane, Phys. Rep. 117, 75 (1985).

[8] S.L. Glashow and S. Weinberg, Phys. Rev. D15, 1958 (1977).

[9] H. Georgi and D.V. Nanopoulos, Phys. Lett. 82B, 95 (1979).

[10] H.E. Haber, G.L. Kane and T. Sterling, Nucl. Phys. B161, 493 (1979).

[11] L.J. Hall and M.B. Wise, Nucl. Phys. B187, 397 (1981).

[12] J.F. Donoghue and L.F. Li, Phys. Rev. D19, 945 (1979).

[13] W.S. Hou, Phys. Lett. B296, 179 (1992); D. Chang, W.S. Hou and W.Y. Keung, Phys. Rev. D48, 217 (1993); D. Atwood, L. Reina and A. Soni, Phys. Rev. D55, 3156 (1997).

[14] For a comprehensive review of the two-Higgs-doublet model, see R.A. Diaz Sanchez, Ph. D. Thesis, Universidad Nacional de Colombia, 2002, arXiv:hep-ph/0212237.

[15] F.J. Botella and J.P. Silva, Phys. Rev. D51, 3870 (1995).

[16] C. Jarlskog, Phys. Rev. Lett. 55, 1039 (1985); Z. Phys. C29, 491 (1985); I. Dunietz, O. W. Greenberg and D.D. Wu, Phys. Rev. Lett. 55, 2935 (1985).

[17] J. Bernabeu, G.C. Branco and M. Gronau, Phys. Lett. B169 (1986) 243; J.D. Bjorken and I. Dunietz, Phys. Rev. D36, 2109 (1987); C. Jarlskog, Phys. Rev. D35, 1685 (1987); D36, 2128 (1987); C. Jarlskog and A. Kleppe, Nucl. Phys. B286, 245 (1987). For a nice review of these techniques, see ref. [6], chapters 14 and 22 .

[18] M. Gronau, A. Kfir and R. Loewy, Phys. Rev. Lett. 56, 1538 (1986); S. Davidson and J.R. Ellis, Phys. Lett. B390, 210 (1997) arXiv:hep-ph/9609451; Phys. Rev. D56, 4182 (1997) arXiv:hep-ph/9702247; S. Davidson, Phys. Lett. B439, 63 (1998) arXiv:hep-ph/9808425; 
J. Ferrandis, Phys. Rev. D60, 095012 (1999) arXiv:hep-ph/9810371; Y. Grossman and H.E. Haber, Phys. Rev. D63, 075011 (2001) arXiv:hep-ph/0005276; S. Davidson and M. Losada, Phys. Rev. D65, 075025 (2002) arXiv:hep-ph/0010325; F.J. Botella, M. Nebot and O. Vives, CERN-PH-TH/2004-138 arXiv:hep-ph/0407349.

[19] L. Lavoura and J.P. Silva, Phys. Rev. D50, 4619 (1994);

[20] I.F. Ginzburg and M. Krawczyk, arXiv:hep-ph/0408011

[21] F. Boudjema and A. Semenov, Phys. Rev. D66, 095007 (2002) arXiv:hep-ph/0201219.

[22] J.F. Donoghue and L.F. Li, Phys. Rev. D19, 945 (1979).

[23] L. Lavoura, Phys. Rev. D50, 7089 (1994) arXiv:hep-ph/9405307.

[24] J.F. Gunion and H.E. Haber, Phys. Rev. D67, 075019 (2003) arXiv:hep-ph/0207010.

[25] J.F. Gunion and H.E. Haber, Phys. Rev. D72, 095002 (2005) arXiv:hep-ph/0506227.

[26] G.C. Branco and M. N. Rebelo, Phys. Lett. B160, 117 (1985).

[27] R. Hempfling, Phys. Rev. D49, 6168 (1994); L.J. Hall, R. Rattazzi and U. Sarid, Phys. Rev. D50, 7048 (1994) arXiv:hep-ph/9306309; M. Carena, M. Olechowski, S. Pokorski and C.E.M. Wagner, Nucl. Phys. B426, 269 (1994) arXiv:hep-ph/9402253 ; J.A. Coarasa, R. A. Jimenez and J. Sola, Phys. Lett. B389, 312 (1996) arXiv:hep-ph/9511402; D.M. Pierce, J.A. Bagger, K.T. Matchev and R.J. Zhang, Nucl. Phys. B491, 3 (1997) arXiv:hep-ph/9606211.

[28] G.C. Branco, M.N. Rebelo and J.I. Silva-Marcos, Phys. Lett. B614, 187 (2005) arXiv:hep-ph/0502118.

[29] P.M. Ferreira, R. Santos and A. Barroso, Phys. Lett. B603, 219 (2004) arXiv:hep-ph/0406231.

[30] A. Santamaria, Phys. Lett. B305, 90 (1993) arXiv:hep-ph/9302301.

[31] S. Dimopoulos and D.W.Sutter, Nucl. Phys. B452, 496 (1995) arXiv:hep-ph/9504415; D.W. Sutter, arXiv:hep-ph/9704390.

[32] See, e.g., R.A. Horn and C.R. Johnson, Matrix Analysis (Cambridge University Press, Cambridge, UK, 1985) pp. 86-87.

[33] S. Weinberg, Phys. Rev. Lett. 40, 223 (1978); F. Wilczek, Phys. Rev. Lett. 40, 279 (1978). 